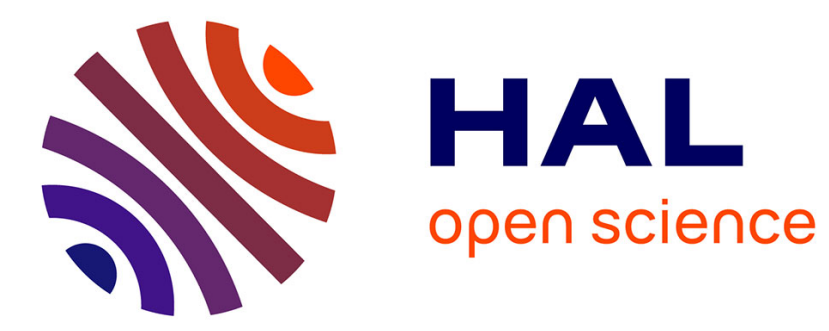

\title{
Improved shunt damping with two negative capacitances: an efficient alternative to resonant shunt
}

Marta Berardengo, Olivier Thomas, Christophe Giraud-Audine, Stefano

Manzoni

\section{- To cite this version:}

Marta Berardengo, Olivier Thomas, Christophe Giraud-Audine, Stefano Manzoni. Improved shunt damping with two negative capacitances: an efficient alternative to resonant shunt. Journal of Intelligent Material Systems and Structures, 2016, pp.1-17. 10.1177/1045389X16667556 . hal-01392769

\section{HAL Id: hal-01392769 \\ https://hal.science/hal-01392769}

Submitted on 4 Nov 2016

HAL is a multi-disciplinary open access archive for the deposit and dissemination of scientific research documents, whether they are published or not. The documents may come from teaching and research institutions in France or abroad, or from public or private research centers.
L'archive ouverte pluridisciplinaire HAL, est destinée au dépôt et à la diffusion de documents scientifiques de niveau recherche, publiés ou non, émanant des établissements d'enseignement et de recherche français ou étrangers, des laboratoires publics ou privés. 


\title{
Improved shunt damping with two negative capacitances: an efficient alternative to resonant shunt
}

\author{
M. Berardengo', O. Thomas ${ }^{2}$, C. Giraud-Audine ${ }^{3}$ and S. Manzoni ${ }^{4}$
}

\begin{abstract}
This paper deals with piezoelectric shunt damping enhanced with negative capacitances. A novel electrical circuit layout is addressed, based on the use of two negative capacitances. It is shown that the shunt performances, in terms of vibration reduction and stability margins, are increased as compared with the classical single negative capacitance layouts. Then, the article focuses on the comparison of a simple resistive shunt, enhanced by a pair of negative capacitances, with a classical resonant shunt. It is shown that the newly proposed enhanced resistive shunt can show equivalent performances in terms of vibration attenuation than the resonant shunt, with at the same time an increased robustness to frequency detuning, in the case of mono-modal damping. The broadband control capability of the resistive shunt coupled to the new negative capacitance layout is also evidenced. The main part of the work is analytical, and then the model is validated by an extensive experimental campaign at the end of the paper.
\end{abstract}

\section{Keywords}

negative capacitance, piezoelectric shunt, resistive shunt, resonant shunt, vibration control, damping, smart structure

\section{Introduction}

Piezoelectric shunt is a well-known technique for vibration damping. Such a method is based on the electrical link between a piezoelectric actuator, bonded to a given vibrating structure, and a properly designed electrical network (Hagood and von Flotow, 1991). Usually, the most used shunt impedances for single mode control are a simple resistance ( $\mathrm{R}$ shunt or resistive shunt) and the series of a resistance and an inductance (LR shunt or resonant shunt) (Hagood and von Flotow, 1991; Thomas et al., 2012; Yamada et al., 2010). The resonant shunt is usually preferred because it offers attenuation levels much higher than the resistive shunt (Hagood and von Flotow, 1991; Thomas et al., 2012) when the parameters of the shunt impedance are perfectly tuned on those of the mechanical eigenmode to damp. On the other hand, the resonant shunt is really sensitive to possible mistunings between the mechanical system and the electric impedance (e.g. due to thermal shifts causing a change of the mechanical eigenfrequency to control) (Berardengo et al., 2015a).

It has been proved in the literature (Thomas et al., 2012) that once the electric impedance is optimally tuned, the performance of the control depends just on the modal electromechanical coupling factor (MEMCF) (Ducarne et al., 2012; Thomas et al., 2009,
2012) of each mode of the electromechanical system (EMS, composed by the vibrating structure, the piezoelectric actuator and the shunt impedance). The MEMCF is a function of the geometrical, mechanical and electrical characteristics of the piezoelectric actuator and the structure, and it is also related to the distance between the natural frequencies of the EMS in short circuit (SC) and open circuit (OC) of the mode considered. The higher the MEMCF is, the higher the maximum achievable attenuation is (Thomas et al., 2012). As mentioned, the value of this parameter is fixed by the kind of structure and the actuator used and therefore also the maximum achievable attenuation level is fixed.

One possibility to improve the damping capability of the shunt control is to use negative capacitances (NCs) in the shunt impedance. Indeed, the use of NCs

\footnotetext{
'Università degli Studi di Parma, Dept. of Industrial Engineering, Parma, Italy

${ }^{2}$ Arts et Métiers ParisTech, LSIS UMR CNRS 7296, Lille, France

${ }^{3}$ Arts et Métiers ParisTech, L2EP, Lille, France

${ }^{4}$ Politecnico di Milano, Dept. of Mechanical Engineering, Milan, Italy
}

\section{Corresponding author:}

Marta Berardengo, Department of Industrial Engineering, Università degli Studi di Parma, Parco Area delle Scienze, I8I/A, Parma, 43 I24, Italy.

Email: marta.berardengo@unipr.it 


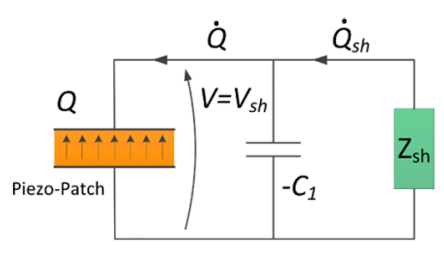

a)

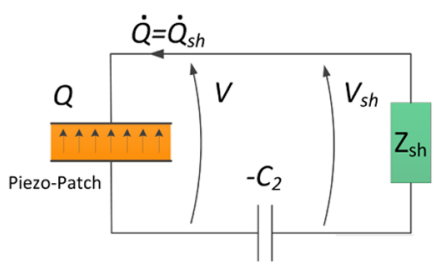

b)

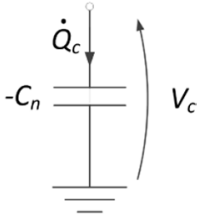

c)

Figure I. Piezoelectric shunt with addition of a NC: parallel configuration (a), series configuration (b) and definition of a NC (c). Here $Z_{\text {sh }}$ is the shunt impedance.

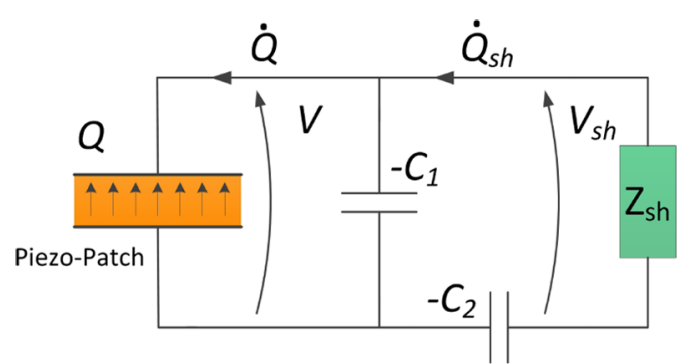

Figure 2. Piezoelectric shunt with addition of two NCs: the newly proposed series + parallel configuration. Here $Z_{\text {sh }}$ is the shunt impedance.

has already been proved to enhance the attenuation provided by resistive (Beck et al., 2013, 2014; Behrens et al., 2003; Berardengo et al., 2016; Collet et al., 2011; de Marneffe and Preumont, 2008; Kodejška et al., 2012; Manzoni et al., 2012; Park and Baz, 2005) and resonant shunts (de Marneffe and Preumont, 2008; Heuss et al., 2014). Indeed, the effect of the NC in the shunt impedance is to artificially increase simultaneously the MEMCF (Berardengo et al., 2016) of all of the modes of an EMS. This effect can be fruitfully employed to improve a lot the attenuation achievable by means of a resistive shunt, keeping at the same time its robustness to possible mistunings. This makes the use of NCs in piezoelectric shunt really attractive. Nevertheless, it has to be remarked that a NC is an active component which does not exist in nature. It can be practically implemented by using a supplied circuit based on an operational amplifier (OP-AMP) (Date et al., 2000; Horowitz and Hill, 1989). This leads to the main drawback of NCs: they can lead to instabilities of the EMS, which need to be addressed.

A NC is traditionally coupled to the piezoelectric patch in parallel or series configuration (see Figure 1(a) and (b)), where the NC (i.e. $-C_{1}$ and $-C_{2}$ for the parallel and the series layout, respectively) is defined as $-C_{n}=Q_{c} / V_{c}$ (see Figure 1(c)). Recently, the authors proposed a new shunt circuit, based on a pair of NCs and able to further increase the attenuation levels provided by the use of a single NC (Berardengo et al., 2015b, 2016). In this new layout, one of the two NCs is connected in parallel (see $-C_{1}$ in Figure 2) and the other in series (see $-C_{2}$ in Figure 2). According to the scheme of Figure 2, the new layout is thus defined as a series + parallel (SP) configuration.

The aim of this paper is to present some new advantages of the newly proposed SP layout. The SP configuration is indeed able to provide higher attenuation levels if compared with the simple series and parallel layouts, but it can also be used to achieve the same attenuation level guaranteeing a higher margin from instability, as demonstrated further in this paper. Furthermore, we will also compare a resistive shunt, enhanced by the SP layout, to the standard resonant shunt. This comparison will address the attenuation levels achievable by means of these shunt layouts as well as their robustness to mistuning.

The paper is structured as follows: the next two sections discuss the model of the EMS used in this work and introduce the use of NCs (and more particularly the SP), investigating its effects on the system. Furthermore, the stability issues and the achievable performances are addressed. Then, we present the advantages provided by the SP configuration if compared with the traditional series and parallel layouts and investigates its level of robustness. Furthermore, we compare the resistive shunt coupled to SP and the simple resonant shunt. Finally, the last section of the paper shows the experimental activity carried out to validate the model of the resistive shunt coupled to SP and its enhanced attenuation performance if compared with the series and parallel layouts.

\section{Model of the electromechanical system}

The model employed to describe the behaviour of the EMS is the one described by Thomas et al. (2012), refined by using a modified value for the piezoelectric patch capacitance, to guarantee a better description of the EMS behaviour in the case of a single degree of freedom approximation, as explained by Berardengo et al. (2016).

If the modal density of the EMS is low, a singledegree-of-freedom approximation can indeed be fruitfully employed to describe the behaviour of the EMS in a frequency band narrowed around the eigenfrequency associated to the mode considered. The original model 


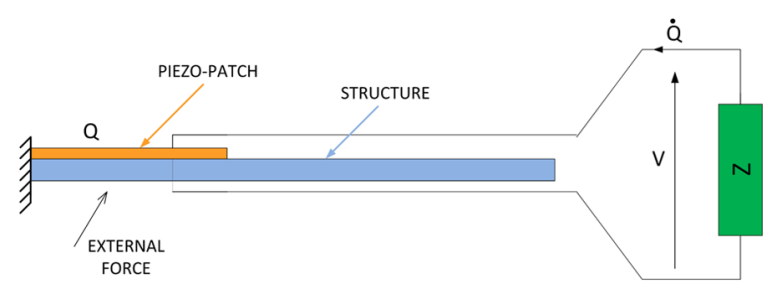

Figure 3. An arbitrary structure with a piezoelectric patch connected to a shunt impedance $Z$.

of Thomas et al. (2012), as well as its improved version for the single-degree-of-freedom approximation, are briefly summarized in this section in order to allow the reader to have a clear understanding of the analytical treatment developed for the new NC layout described in the following.

A generic elastic structure with one piezoelectric patch bonded on it and excited by an external force $F$ is taken into account (see Figure 3). $U(x, t)$ is the displacement of any point $x$ of the structure at time $t$. An arbitrary shunt impedance $Z$ is connected to the piezoelectric patch and $V$ is the voltage between the electrodes of the piezoelectric actuator; $Q$ is the electric charge in one of the electrodes and, considering the convention of sign for $V$ in Figure 3, $Q$ is precisely the charge in the upper electrode. A reduced order model of the EMS can be obtained by expressing the displacement $U$ in modal coordinates and considering $N$ (theoretically $N \rightarrow+\infty$ ) vibration eigenmodes:

$$
U(x, t)=\sum_{i=1}^{N} \Phi_{i}(x) q_{i}(t)
$$

where $q_{i}$ is the $i$ th modal coordinate and $\Phi_{i}$ is the $i$ th eigenmode of the structure (normalized to the unit modal mass). If a single-degree-of-freedom approximation is considered by keeping only the $i$ th mode in the modal truncation, the behaviour of the EMS can be described for $\Omega \simeq \omega_{i}$ (where $\Omega$ is the angular frequency) as

$$
\begin{gathered}
\ddot{q}_{i}+2 \xi_{i} \omega_{i} \dot{q}_{i}+\omega_{i}^{2} q_{i}-\chi_{i} V=F_{i} \\
C_{p i} V-Q+\chi_{i} q_{i}=0
\end{gathered}
$$

where Equation (2) is the equation of motion of the EMS and it is coupled to the EMS electrical behaviour, described by Equation (3), by means of the coefficient $\chi_{i} . F_{i}$ is the modal forcing, that depends on $F$ and the $i$ th mode shape $\Phi_{i}$. Here $\omega_{i}$ is the $i$ th natural frequency of the EMS in short circuit, $\xi_{i}$ is the $i$ th structural damping factor and $\chi_{i}$ is a modal coupling coefficient, which is related to the energy transfer between the $i$ th mode shape and the piezoelectric patch. The values of $\chi_{i}$ depend on the geometrical, electrical and mechanical characteristics of the piezoelectric actuator and the structure, and on the position of the actuator. Those $\chi_{i}$ coefficients can be computed with either an analytical model (Ducarne et al., 2012) or by a finite element discretization (Thomas et al., 2009). It should be noted that $\Phi_{i}$ are the eigenmodes of the EMS with the piezoelectric patch in $\mathrm{SC}$ (with $V=0$ ).

The term $C_{p i}$ in Equation (3) is the value of the capacitance of the piezoelectric patch at frequencies above $\omega_{i}$ when a single-degree-of-freedom approximation is performed on the complete model, and it is defined as

$$
C_{p i}=C_{\infty}+\sum_{n=i+1}^{N} \frac{\chi_{n}^{2}}{\omega_{n}^{2}}
$$

where $C_{\infty}$ is the electrical blocked capacitance of the piezoelectric patch (with $U(x, t)=0 \forall x \Rightarrow q_{i}=0 \forall i$ ). The second term of the right-hand side of Equation (4) accounts for the contribution to the capacitance value of the modes higher than the $i$ th, which are neglected in the single-degree-of-freedom approximation. This static correction, introduced in Berardengo et al. (2016), is an improvement to the model proposed in Thomas et al. (2012) and allows us to obtain a better description of the EMS when a single-degree-of-freedom approximation is taken into account. The value of $C_{p i}$ can be estimated by measuring the capacitance of the piezoelectric patch between the $i$ th and $(i+1)$ th modes. More details about the definition of $C_{p i}$ and its estimation can be found in Berardengo et al. (2016).

The model described by Equations (2) and (3) is related to the case of a simple piezoelectric shunt where no NC is included in the circuit. Now the addition of NCs is addressed, according to the schemes of Figures 1 and 2. Following the procedure described in Berardengo et al. (2016), it is convenient to apply the change of variables:

$$
\bar{V}_{s h}=V_{s h} \sqrt{C_{e q}}, \quad \bar{Q}_{s h}=\frac{Q_{s h}}{\sqrt{C_{e q}}}
$$

where $V_{s h}$ and $Q_{s h}$ are the voltage and the charge seen by the shunt impedance $Z_{s h}$, and $C_{e q}$ is an equivalent capacitance, defined in Table 1 . Such an equivalent capacitance depends on the NC connected to the capacitance of the piezoelectric patch, and thus depends on the type of connection (parallel, series or SP). Substituting Equation (5) in Equations (2) and (3), the following system of equations is obtained:

$$
\begin{gathered}
\ddot{q}_{i}+2 \xi_{i} \omega_{i} \dot{q}_{i}+\left(\omega_{i}^{\mathrm{sc}}\right)^{2} q_{i}-\omega_{i} \tilde{k}_{i} \bar{V}_{\mathrm{sh}}=F_{i} \\
\ddot{q}_{i}+2 \xi_{i} \omega_{i} \dot{q}_{i}+\left(\omega_{i}^{\mathrm{oc}}\right)^{2} q_{i}-\omega_{i} \tilde{k}_{i} \bar{Q}_{\mathrm{sh}}=F_{i} \\
\bar{V}_{\mathrm{sh}}-\bar{Q}_{\mathrm{sh}}+\omega_{i} \tilde{k}_{i} q_{i}=0
\end{gathered}
$$

It should be noted that Equations (6) and (7) play the same role: one is expressed as function of $\bar{V}_{s h}$ (i.e. Equation (6)) and the other as function of $\bar{Q}_{s h}$ (i.e. 
Table I. Parameters of the EMS enhanced by a single NC in parallel and series configuration and by two NCs for the series + parallel (SP) configuration.

\begin{tabular}{|c|c|c|c|c|}
\hline & & Parallel configuration & Series configuration & SP configuration \\
\hline SC frequency & $\omega_{i}^{s c}$ & $\omega_{i}$ & $\sqrt{\omega_{i}^{2}-\frac{\chi_{i}^{2}}{C_{2}-C_{p i}}}$ & $\sqrt{\omega_{i}^{2}-\frac{\chi_{i}^{2}}{C_{1}+C_{2}-C_{\mathrm{p} i}}}$ \\
\hline OC frequency & $\omega_{i}^{o c}$ & $\sqrt{\omega_{i}^{2}+\frac{\chi_{i}^{2}}{C_{p i}-C_{1}}}$ & $\hat{\omega}_{i}=\sqrt{\omega_{i}^{2}+\frac{\chi_{i}^{2}}{C_{\mathrm{p} i}}}$ & $\sqrt{\omega_{i}^{2}+\frac{\chi_{i}^{2}}{C_{p i}-C_{1}}}$ \\
\hline Equivalent capacitance & $C_{\text {eq }}$ & $C_{p i}-C_{1}$ & $\frac{C_{p i} C_{2}}{C_{2}-C_{p i}}$ & $\frac{\left(C_{p i}-C_{1}\right) C_{2}}{C_{1}+C_{2}-C_{p i}}$ \\
\hline EMEMCF & $\tilde{k}_{i}$ & $\frac{k_{i}}{c}$ & $\frac{k_{i}}{c}$ & $\frac{k_{i}}{{ }_{1}}$ \\
\hline
\end{tabular}

Equation (7)). Here $\omega_{i}^{\mathrm{sc}}$ and $\omega_{i}^{\mathrm{oc}}$ are respectively the natural frequency of the EMS with the shunt shortcircuited $\left(V_{\mathrm{sh}}=0\right)$ or in open circuit $\left(Q_{\mathrm{sh}}=0\right)$, defined in Table 1. It should be noted that their values depend on the NCs $C_{1}$ and $C_{2}$. The term $\tilde{k}_{i}$, which appears naturally in the system, is defined as the enhanced modal electromechanical coupling factor (EMEMCF) and assumes the following expression:

$$
\tilde{k}_{i}=\sqrt{\frac{\left(\omega_{i}^{o c}\right)^{2}-\left(\omega_{i}^{s c}\right)^{2}}{\omega_{i}^{2}}}
$$

We can observe that if we fix $\omega_{i}^{s c}=\omega_{i}$ and $\omega_{i}^{o c}=\hat{\omega}_{i}$ (where $\hat{\omega}_{i}$ and $\omega_{i}$ are the OC and SC eigenfrequency respectively of the EMS without the addition of any NCs), we obtain the classical expression of the MEMCF $k_{i}$ (which is close to the $i$ th effective coupling factor) (Berardengo et al., 2016; Thomas et al., 2012):

$$
k_{i}=\frac{\chi_{i}}{\omega_{i} \sqrt{C_{p i}}}=\sqrt{\frac{\left(\hat{\omega}_{i}\right)^{2}-\left(\omega_{i}\right)^{2}}{\omega_{i}^{2}}}
$$

Therefore the EMEMCF is a parameter analogous to the MEMCF, but enhanced by the presence of the NCs. The analytical formulations of all of these parameters involved in the definition of the EMEMCF $\tilde{k}_{i}$ depend on the layout used to connect the NCs and they are gathered in Table 1.

\section{Enhanced resistive shunt}

\section{Model}

If a simple resistance $R_{s h}$ is used as the shunt impedance $Z_{s h}$ in Figures 1 and 2, one has

$$
V_{s h}=-R_{s h} \dot{Q}_{s h} \quad \Rightarrow \quad \bar{V}_{s h}=-\tau_{e} \dot{\bar{Q}}_{s h} \quad \text { with } \tau_{e}=R_{s h} C_{e q}
$$

where $\tau_{e}$ is the electric time constant and where the change of variables of Equation (5) has been used. Using Equations (6), (7) and (8), the following frequency response function (FRF) can be defined (Berardengo et al., 2016):

$$
\begin{aligned}
& H_{i}(\Omega)=\frac{q_{i}}{F_{i}}= \\
& \frac{1+j \tau_{e} \Omega}{\left(\omega_{i}^{\mathrm{sc}}\right)^{2}-\left(1+2 \xi_{i} \omega_{i} \tau_{e}\right) \Omega^{2}+j \Omega\left[\tau_{e}\left(\omega_{i}^{\mathrm{oc}}\right)^{2}+2 \xi_{i} \omega_{i}-\tau_{e} \Omega^{2}\right]}
\end{aligned}
$$

where $j$ is the imaginary unit.

According to Berardengo et al. (2016), the analytical expression of this FRF is the same of that describing a pure resistive shunt without NCs added in the circuit (Thomas et al., 2012), except that one has to use the proper value of the parameters in Equation (12): $\omega_{i}^{s c}=\omega_{i}, \omega_{i}^{o c}=\hat{\omega}_{i}$ and $C_{e q}=C_{p i}$. Thanks to this equivalence between the analytical representations of the EMS with and without NCs, the same procedure presented in Thomas et al. (2012) for the optimal tuning of $R_{s h}$ and for the estimation of the attenuation performance can be used here. This procedure allows us to define the optimal value of $\tau_{e}$ (named $\tau_{e}^{\text {opt }}$ ), and thus of $R_{s h}$, which minimizes the resonance peak amplitude at given mode, as

$$
\tau_{e}^{o p t}=\sqrt{\frac{2}{\left(\omega_{i}^{o c}\right)^{2}+\left(\omega_{i}^{s c}\right)^{2}}} \simeq \frac{1}{\omega_{i}} \sqrt{\frac{2}{\tilde{k}_{i}^{2}+2}}
$$

It depends only on the EMEMCF $\tilde{k}_{i}$ and on the natural frequency of the targeted mode.

The performances of the present enhanced resistive shunt are evaluated by the resonance peak attenuation parameter $A_{\mathrm{dB}}$, measured at the resonance of the mode on which $\tau_{e}$ has been tuned, defined as (Berardengo et al., 2016)

$$
A_{\mathrm{dB}}=20 \log _{10} \frac{H_{\mathrm{sc}}}{H_{\mathrm{sh}}} \simeq 20 \log _{10} \frac{\tilde{k}_{i}^{2}+2 \sqrt{2} \xi_{i} \sqrt{2+\tilde{k}_{i}^{2}}}{4 \xi_{i} \sqrt{1-\xi_{i}^{2}}}
$$

where $H_{\mathrm{sc}}$ is the FRF peak amplitude of the EMS in SC and without NCs (i.e. uncontrolled EMS), while $H_{\text {sh }}$ is 


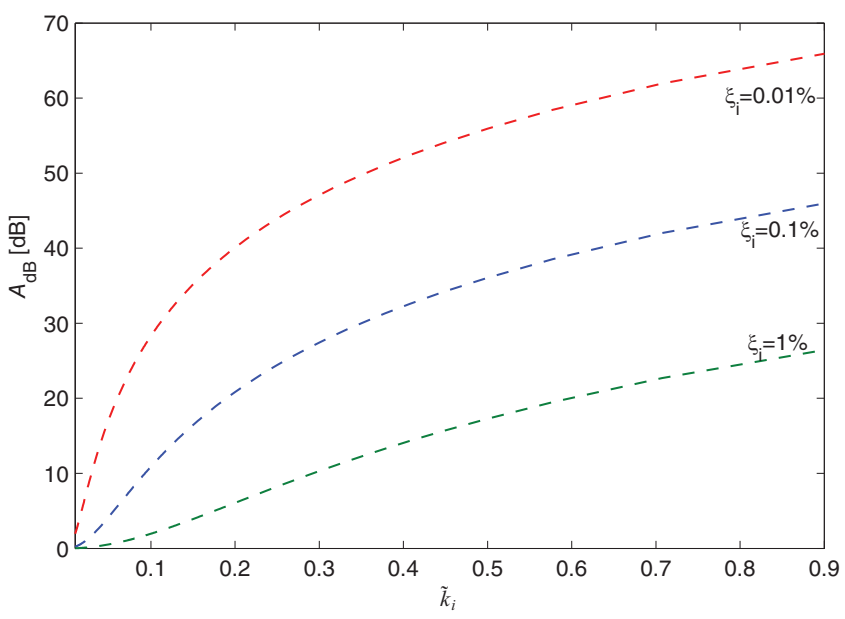

Figure 4. Trend of $A_{\mathrm{dB}}$ (see Equation (14)) as a function of $\tilde{k}_{i}$ for different values of $\xi_{\text {i }}$.

the peak amplitude of the FRF when the optimized shunt is employed (i.e. $\tau_{e}=\tau_{e}^{o p t}$ ). The performance of the control (measured by $A_{\mathrm{dB}}$ ) depends only on the structural damping $\xi_{i}$ and on the EMEMCF $\tilde{k}_{i}$ of the targeted mode, as illustrated in Figure 4; it increases as a function of $\tilde{k}_{i}$.

\section{Stability of the negative capacitance}

As mentioned previously, the $\mathrm{NC}$ is an active component which can be built in practice using an OP-AMP. The use of active components in the shunt impedance makes the control strategy no more passive. Therefore, the study of the stability of the system is mandatory. To this purpose, the Routh-Hurwitz criterion (Gopal, 2002) has been applied to the FRF of Equation (12) and the stability conditions are found to be

$$
\begin{array}{ll}
\text { parallel : } & C_{1}<C_{p i} \\
\text { series : } & C_{2}>C_{p i}\left(1+k_{i}^{2}\right) \\
\text { SP : } & C_{1}<C_{p i} \quad \& \quad C_{2}+C_{1}>C_{p i}\left(1+k_{i}^{2}\right)
\end{array}
$$

It should be noted that there are two stability conditions for the SP because two NCs are used together.

Since Equation (12) is the EMS FRF when a single degree of freedom is considered, Equation (15) gives the stability limits associated just to the mode taken into account. However, when a stability analysis is carried out, the whole EMS must be taken into account in order to avoid problems due to spillover (Burns, 2001). Therefore, the stability conditions related to the EMS are the strictest ones found by applying the RouthHurwitz criterion to all of the modes of the EMS. Such stability conditions are found to be

$$
\begin{array}{lll}
\text { parallel : } & C_{1}<C_{\infty} \\
\text { series : } & C_{2}>C_{0} \\
\text { SP : } & C_{1}<C_{\infty} \quad \& \quad C_{2}+C_{1}>C_{0}
\end{array}
$$

where $C_{0}=C_{p 1}\left(1+k_{1}^{2}\right)$ is the value of the capacitance of the piezoelectric patch at the null frequency (Berardengo et al., 2016). It should be noted that the conditions of Equation (16) depend on all of the modes of the structure.

It is worth highlighting that when the stability conditions of Equation (16) are fulfilled, the value of $\tilde{k}_{i}$ is bigger than the original MEMCF $k_{i}$ (see Table 1) and its value depends on the value of the $\mathrm{NC}$ which is used: the closer the $\mathrm{NC}$ is to the stability limit, the higher the value of $\tilde{k}_{i}$ is.

Table 1 provides evidence of another interesting aspect related to the use of a $\mathrm{NC}$. The $\mathrm{NC}$ in parallel configuration does not change the SC eigenfrequency (i.e. the $\mathrm{SC}$ eigenfrequency is the same with and without $\mathrm{NC}$ ), while the OC eigenfrequency is shifted. If the condition of Equation (16) for the parallel layout is fulfilled, the OC eigenfrequency increases thanks to the use of the NC. This also explains the reason why the value of $\tilde{k}_{i}$ is increased if compared with $k_{i}$ : the SC and OC eigenfrequencies are farther than in the case of absence of NC. The series configuration of the $\mathrm{NC}$ again makes the $\mathrm{SC}$ and $\mathrm{OC}$ eigenfrequencies to be farther than in the case of absence of the NC. This time the OC eigenfrequency does not change adding or removing the $\mathrm{NC}$, but the SC eigenfrequency is shifted towards lower frequency values. Now, if we consider the SP configuration, we note that the $\mathrm{SC}$ and $\mathrm{OC}$ eigenfrequencies are both shifted at the same time: the SC towards lower frequency values and the OC towards higher frequency values, and this explains why the SP is able to increase the value of $\tilde{k}_{i}$ if compared with the traditional series and parallel layouts (see the next section).

The SP configuration could be coupled to whatever kind of shunt impedance (e.g. a LR shunt) because the effect on the value of $\tilde{k}_{i}$ is not related to the layout of $Z_{s h}$. Previously in this section we have considered a simple resistance as shunt impedance. Usually, the pure resistive shunt is avoided due to its low attenuation performance but one of the goal of this paper is to show that the enhancement of the MEMCF provided by the SP NC is able to make this kind of shunt really competitive, keeping at the same time its advantages: ease of use, robustness to uncertainty and biases, and capability to damp many modes together on a wide frequency range.

\section{Advantages of the resistive series + parallel configuration}

In this section the advantages provided by the use of the SP configuration in terms of performances and robustness are evidenced and its behaviour is compared with that of traditional NC layouts: series and parallel. To this purpose, it is convenient to introduce four indexes: 
Table 2. EMEMCF as a function of the parameters $\beta$.

\begin{tabular}{|c|c|c|c|c|c|}
\hline & & R shunt & Parallel configuration & Series configuration & SP configuration \\
\hline \multirow[t]{2}{*}{ EMEMCF } & $\tilde{k}_{i}$ & $k_{i}$ & $k_{i}$ & $k_{i}$ & $k_{i}$ \\
\hline & & & $\sqrt{\sqrt{I-\beta_{I}}}$ & $\sqrt{\sqrt{I-\beta_{2}}}$ & $\sqrt{\frac{\left(1-\beta_{s p 1}\right)\left(I-\beta_{s p 22}\right)}{I-\beta_{s p 1} \beta_{s p 2}}}$ \\
\hline
\end{tabular}

$\beta_{1}=\frac{C_{1}}{C_{\mathrm{p} i}}, \quad \beta_{2}=\frac{C_{\mathrm{p} i}}{C_{2}}, \quad \beta_{\mathrm{sp} 1}=\frac{C_{1}}{C_{\mathrm{p} i}}, \quad \beta_{\mathrm{sp} 2}=\frac{C_{\mathrm{p} i}}{C_{1}+C_{2}}$

where $\beta_{1}$ is related to the parallel configuration, $\beta_{2}$ to the series, and $\beta_{s p 1}$ and $\beta_{s p 2}$ to the SP configuration. These indices are positive and define how much the NCs are close to the value of $C_{p i}$ : the closer the $\beta$ indexes are to 1 , the closer the NCs are to $C_{p i}$. Null values of $\beta$ means, instead, absence of the NCs (i.e. simple piezoelectric shunt). It should be noted that the $\beta$ coefficients are strictly smaller than the value of 1 because in this case the NC value would be over the instability threshold (see Equation (16)). According to Equation (17) and to Table 1 , the EMEMCF $\tilde{k}_{i}$ can be defined also as function of the $\beta$ coefficients as shown in Table 2.

\section{Enhancement of attenuation and stability margins}

The expressions of the EMEMCF in Tables 1 and 2 allow us to highlight that when the values of the $\beta$ coefficients increase, the values of the EMEMCF increase as well: the closer the NCs are to the stability limits, the higher the value of $\tilde{k}_{i}$ is. This increase of $\tilde{k}_{i}$ leads to an increase of the attenuation $A_{\mathrm{dB}}$ (see Equation (14)) and explains why the shunt performances are improved.

Despite the stability limits of Equation (16) imply that values of $\beta$ equal to 1 cannot be reached, it is still possible to achieve high values of $\beta$ and thus of $\tilde{k}_{i}$. To measure the coupling factor enhancement, we define the following quantity:

$$
G=\tilde{k}_{i} / k_{i}
$$

Figure 5 shows that, given a fixed value of $\beta$ $\left(\beta_{1}=\beta_{2}=\beta_{s p 1}=\beta_{s p 2}\right)$, the SP configuration enables us to obtain higher values of $\tilde{k}_{i}$ if compared with the series and parallel layouts (these curves are obtained using the expressions of Table 2). This is a remarkable result since, according to Equation (14) and Figure 4, this implies that the SP configuration allows to have higher attenuation levels. Therefore, the SP layout is able to enhance the attenuation levels achievable by means of the traditional series and parallel layouts.

As already mentioned, it is not possible to pull the $\beta$ values to 1 because the stability limits discussed previously must be taken into account. Hence, there exists an unstable area, as shown in grey in Figure 5, whose

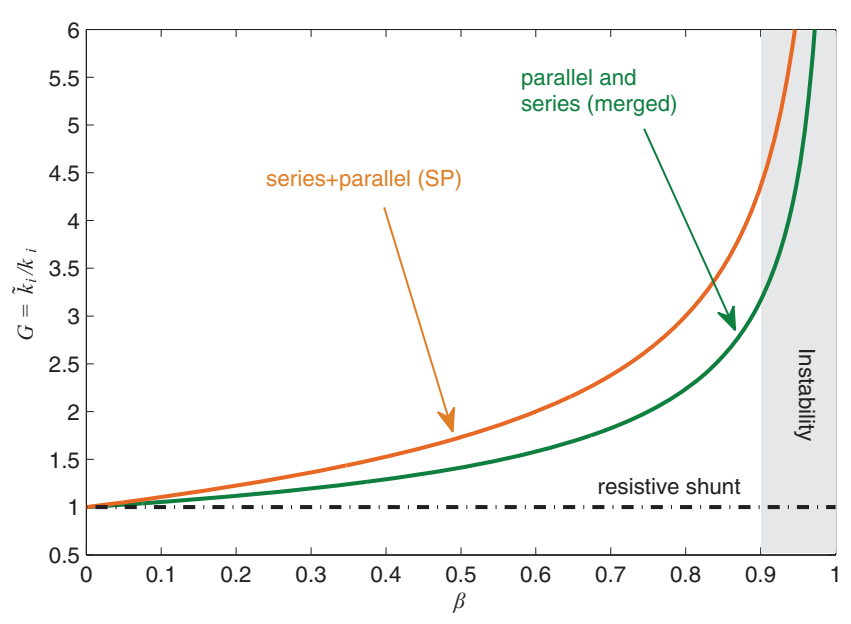

Figure 5. Trend of $G$ as function of $\beta$ (with $\beta=\beta_{\text {I }}=$ $\beta_{2}=\beta_{s p 1}=\beta_{s p 2}$ ) for different negative capacitance layouts: parallel (Figure I (a)), series (Figure I (b)) and SP (Figure 2).

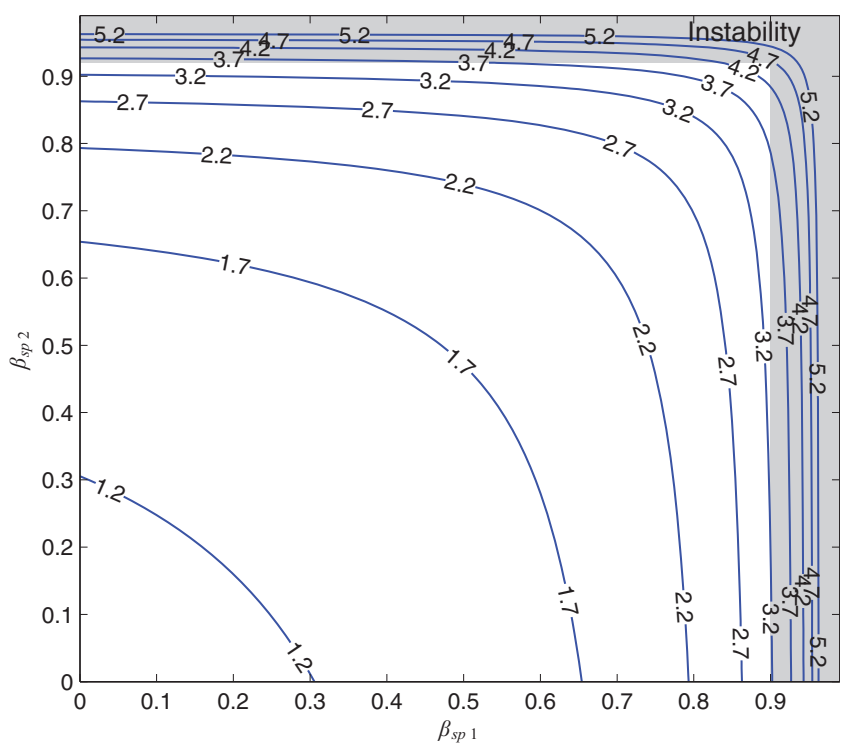

Figure 6. Relationship between $\beta_{s p 1}$ and $\beta_{s p 2}$ providing given values of $G$.

extension depends upon the system and the mode taken into account (Berardengo et al., 2016).

The same considerations can be drawn by looking at Figure 6 where the $G$ value for the SP configuration (last column of Table 2) is shown in isolines as a function of $\beta_{s p 1}$ and $\beta_{s p 2}$. This plot also shows the $G$ values 
achievable with the $\mathrm{NC}$ in parallel (values for $\beta_{s p 2}=0$ ) and in series (values for $\beta_{s p 1}=0$ ) configurations as well, thus leading to a more general and detailed comparison.

It should be noted that the value of $G$ increases in the areas of the figure far from the plot axes, thus highlighting the benefits provided by the SP configuration. To illustrate this point, consider, as an example, a series or parallel NC configuration with $G=1.7$. This value of $G$ is achieved by using $\beta_{2} \simeq 0.65$ for the series and $\beta_{1} \simeq 0.65$ for the parallel (see Table 2). These values of $\beta_{2}$ and $\beta_{1}$ can be found also by looking at the intersection points between the isoline with a value of $G$ equal to 1.7 and the axes in Figure 6. If we use a SP configuration with $\beta_{s p 1}=\beta_{s p 2}=0.65$ (thus we move away from the figure axes), we obtain a higher $G$ value: about 2.17 .

Figure 6 provides evidence that the SP configuration can be employed even to achieve another remarkable goal: it is able to provide the same value of $\tilde{k}_{i}$, and thus of the attenuation $A_{\mathrm{d} B}$, by employing lower values of the $\beta$ coefficients with respect to the simple parallel and series layouts. This allows to use $\mathrm{NC}$ values which guarantee a higher margin from the instability limits. Indeed, the isolines of Figure 6 show that the pairs of $\beta_{s p 1}$ and $\beta_{s p 2}$ which allow us to have the same $G$ value than that achievable with a given value of $\beta_{1}$ or $\beta_{2}$ are such that $\beta_{s p 1}$ and $\beta_{s p 2}$ are lower than $\beta_{1}$ or $\beta_{2}$. This is a remarkable result especially when structures with low values of $k_{i}$ (e.g. thick metal structures) are taken into account. In these cases, the value of the $\mathrm{NC}$ must be very close to the instability in order to achieve a good attenuation level, thus leading to more risky working conditions. The use of SP allows us instead to achieve such a high level of attenuation with a higher reliability if compared with the series and the parallel configurations. Indeed, if a shift of some of the EMS parameters occurs (e.g. due to thermal shifts), the SP turns out to be more reliable than the traditional configurations because of the lower values of the $\beta$ coefficients which can be employed, thus showing higher robustness in terms of stability.

\section{Robustness of the resistive shunt coupled to series + parallel NCs}

An important feature of every control system is its capability to be robust against uncertainties and system changes. Indeed, as an example, a thermal shift could cause a change of the eigenfrequency associated to the mode to control, thus leading to a mistuning and then to the worsening of the attenuation performance. Therefore, in order to have a full understanding of the piezoelectric shunt effectiveness, even the EMS behaviour in mistuned conditions must be taken into account. To this purpose, the robustness of the resistive shunt coupled to the SP layout has been studied by
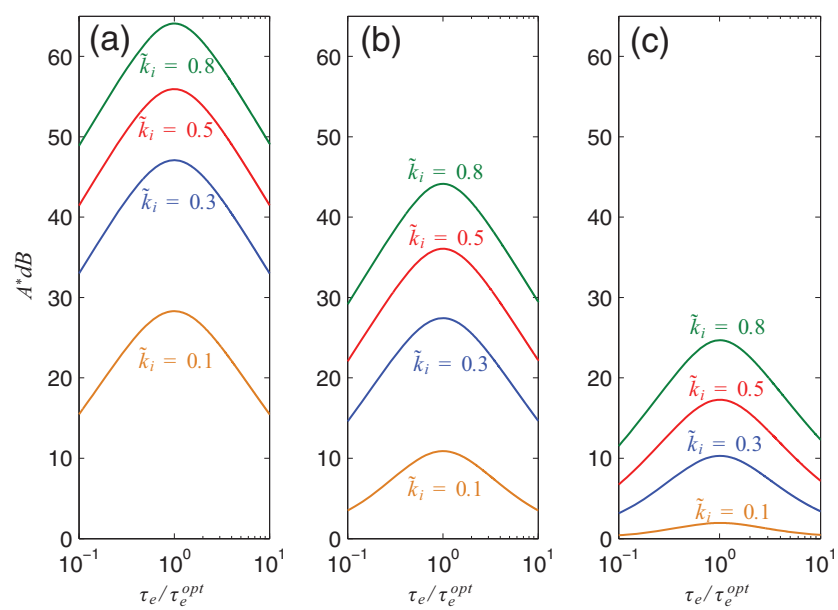

Figure 7. Trend of $A_{\mathrm{dB}}^{*}$ as a function of $\tau_{\mathrm{e}} / \tau_{\mathrm{e}}^{\text {opt }}$ for different values of $\tilde{k}_{i}: \xi_{i}=10^{-4}(\mathrm{a}), \xi_{i}=10^{-3}$ (b) and $\xi_{i}=10^{-2}$ (c).

computing the attenuation $A_{\mathrm{dB}}^{*}$ in mistuned conditions. This latter index is the attenuation provided by the shunt when a value of $\tau_{e} \neq \tau_{e}^{o p t}$ is used. Here $A_{\mathrm{dB}}^{*}$ is defined as

$$
A_{\mathrm{dB}}^{*}=20 \log _{10} \frac{H_{\mathrm{sc}}}{H^{\max }}
$$

where $H^{\max }$ is the FRF peak of the EMS with $\tau_{e} \neq \tau_{e}^{o p t}$, computed numerically by means of Equation (12).

The use of a generic value of $\tau_{e} \neq \tau_{e}^{o p t}$ is representative of a mistuned working condition where the shunt is mistuned. This situation can occur if the shunt is not well tuned or if the natural frequency of the targeted mode has changed as compared with its reference value $\omega_{i}$ used for the tuning of the shunt. Since $\tau_{e}^{\text {opt }}$ is inversely proportional to $\omega_{i}$ (see Equation (13)) and if we denote by $\omega_{i}^{*}$ the current value of the natural frequency, one has $\tau_{e} / \tau_{e}^{\text {opt }}=\omega_{i}^{*} / \omega_{i}$.

Figure 7 shows $A_{\mathrm{dB}}^{*}$ for different values of $\xi_{i}$ and $\tilde{k}_{i}$ (it should be noted that the value of $A_{\mathrm{dB}}^{*}$ is equal to $A_{\mathrm{d} B}$ when $\tau_{e}=\tau_{e}^{o p t}$, compare Figure 7 and Figure 4). It is evident that the higher $\tilde{k}_{i}$ is, the higher the attenuation is, even in mistuned conditions. Indeed, the same value of $\tau_{e} / \tau_{e}^{\text {opt }}$ guarantees higher attenuation levels when $\tilde{k}_{i}$ is high than in the case it is low. Therefore, the increase of $\tilde{k}_{i}$ accomplished by using the SP configuration allows us to assure a high level of attenuation even in presence of mistuning. This means that the use of the SP layout is advantageous if compared with the simple parallel and series NC configurations because it allows us to achieve higher increases of the value of $\tilde{k}_{i}$.

Figure 8 shows the same curves of Figure 7 shifted downwards by a quantity equal to the corresponding optimal attenuation value $A_{\mathrm{dB}}$, and therefore such curves show the loss of attenuation. It is noteworthy to underline that the loss of performance does not depend on the value of $\tilde{k}_{i}$ and $\xi_{i}$ when the value of $A_{\mathrm{dB}}^{*}$ is high enough 

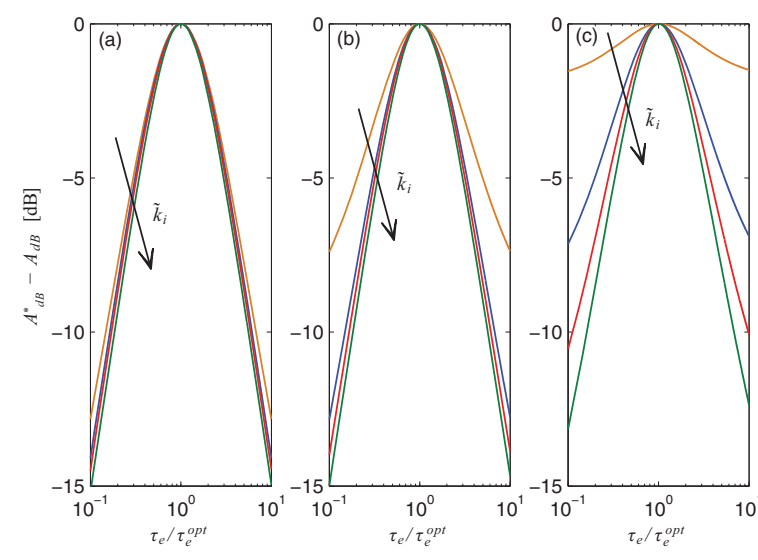

Figure 8. Trend of $A_{\mathrm{dB}}^{*}-A_{\mathrm{dB}}$ as a function of $\tau_{\mathrm{e}} / \tau_{\mathrm{e}}^{\text {opt }}$ for different values of $\tilde{k}_{i}$ (i.e. $0.1,0.3,0.5$ and 0.8 ): $\xi_{i}=10^{-4}$ (a), $\xi_{i}=10^{-3}(\mathrm{~b})$ and $\xi_{i}=10^{-2}(\mathrm{c})$.

(higher than about $10 \mathrm{~dB}$ ), since in this case all of the curves of the three plots of Figure 8 are almost superimposed. This means that the percentage loss of performance depends just on the percentage mistuning on $\tau_{e}$ (i.e. $\left.\tau_{e} / \tau_{e}^{o p t}\right)$. When the attenuation is lower than about $10 \mathrm{~dB}$, the plots of Figure 8 show a change of behaviour (e.g. the curve for $\tilde{k}_{i}=0.1$ in Figure 8(b)) but it is still possible to notice that the features of the system have no influence on the trend of the loss of attenuation. Indeed, the curve with $\tilde{k}_{i}=0.1$ in Figure $7(\mathrm{~b})$ and that with $\tilde{k}_{i}=0.3$ in Figure 7(c) show almost the same value of the optimized attenuation $A_{\mathrm{dB}}$ and their trends for $\tau_{e} \neq \tau_{e}^{o p t}$ are almost equal (the curve with $\tilde{k}_{i}=0.1$ in Figure 8(b) and that with $\tilde{k}_{i}=0.3$ in Figure $8(\mathrm{c})$ match if plotted on the same graph).

resonant circuit together with the capacitance $C_{p i}$ of the piezoelectric patch. Therefore, the LR shunt can be considered as the electrical equivalent of the mechanical tuned mass damper (Preumont, 2011) and thus as a result it is really effective for mono-modal control. We consider here a LR shunt directly coupled to the piezoelectric element with no $\mathrm{NC}$ enhancement $\left(V=V_{s h}\right.$, $\left.Q=Q_{s h}, \tilde{k}_{i}=k_{i}\right)$. In this case, the following relation holds:

$$
V_{s h}=-R_{e} \dot{Q}_{s h}-L_{e} \ddot{Q}_{s h}
$$

Using the change of variables of Equation (5) with $C_{e q}=C_{p i}$ and introducing it in Equation (8), the latter is replaced by

$$
\frac{1}{\omega_{e}^{2}} \ddot{\bar{Q}}_{s h}+\frac{2 \xi_{e}}{\omega_{e}} \dot{\bar{Q}}_{s h}+\bar{Q}_{s h}-\omega_{i} k_{i} q_{i}=0
$$

where $\omega_{e}$ is the electrical angular frequency and $\xi_{e}$ the electrical damping ratio, defined by

$$
\omega_{e}=\frac{1}{\sqrt{L_{e} C_{p i}}}, \quad \xi_{e}=\frac{R_{e}}{2} \sqrt{\frac{C_{p i}}{L_{e}}}
$$

Their optimal values for single mode control have been investigated in Thomas et al. (2012) and read

$$
\omega_{e}^{o p t}=\hat{\omega}_{i}=\omega_{i} \sqrt{1+k_{i}^{2}}, \quad \xi_{e}^{o p t}=k_{i} \sqrt{\frac{3}{8}}
$$

The expression of the FRF $q_{i} / F_{i}$ obtained by using Equations (2), (3) and (21) is (Thomas et al., 2012)

$$
H_{i}^{L R}(\Omega)=\frac{q_{i}}{F_{i}}=\frac{1-\frac{\Omega^{2}}{\omega_{e}^{2}}+2 j \xi_{e} \frac{\Omega}{\omega_{e}}}{\frac{\Omega^{4}}{\omega_{e}^{2}}-\Omega^{2}\left(1+\frac{\hat{\omega}_{i}^{2}}{\omega_{e}^{2}}+4 \xi_{i} \xi_{e} \frac{\omega_{i}}{\omega_{e}}\right)+\omega_{i}^{2}+2 j \Omega\left[\frac{\xi_{e}}{\omega_{e}}\left(\hat{\omega}_{i}^{2}-\Omega^{2}\right)+\xi_{i} \omega_{i}\left(1-\frac{\Omega^{2}}{\omega_{e}^{2}}\right)\right]}
$$

The benefits provided by the use of NCs with SP configuration have been discussed so far. Now, the resistive shunt coupled to SP (RSP in the following) is compared to the shunt impedance mostly employed for single mode damping: the LR shunt. This comparison is addressed in the next section.

\section{Comparison between RSP and LR shunt}

The most frequently used shunt impedance for monomodal attenuation is the LR shunt. This section focuses on the comparison between this well-established approach and the newly proposed RSP shunt.

\section{Resonant shunt model}

The LR shunt is composed by the series of a resistance $R_{e}$ and an inductance $L_{e}$. These two elements form a
Finally, according to Thomas et al. (2012), the attenuation provided by the optimized LR shunt, named here $A_{\mathrm{dB}}^{L R}$, can be computed as

$$
A_{\mathrm{dB}}^{L R}=10 \log _{10} \frac{\operatorname{num}\left(k_{i}, \xi_{i}\right)}{8\left(6 k_{i}^{2}+8-3 k_{i} \sqrt{2\left(1+k_{i}^{2}\right)}\right) \xi_{i}^{2}\left(1-\xi_{i}^{2}\right)}
$$

where

$$
\begin{aligned}
\operatorname{num}\left(k_{i}, \xi_{i}\right) & =8\left[9 k_{i}^{4}+17 k_{i}^{2}+8-k_{i} \sqrt{2\left(1+k_{i}^{2}\right)}\left(6 k_{i}^{2}+7\right)\right] \xi_{i}^{2} \\
& +16 \sqrt{3} k_{i}\left(1+k_{i}^{2}\right)\left(\sqrt{2\left(1+k_{i}^{2}\right)}-k_{i}\right) \xi_{i} \\
& \left.-3 k_{i}^{3} \sqrt{2\left(1+k_{i}^{2}\right)(1}+k_{i}^{2}\right)+6 k_{i}^{6}+14 k_{i}^{4}+8 k_{i}^{2}
\end{aligned}
$$




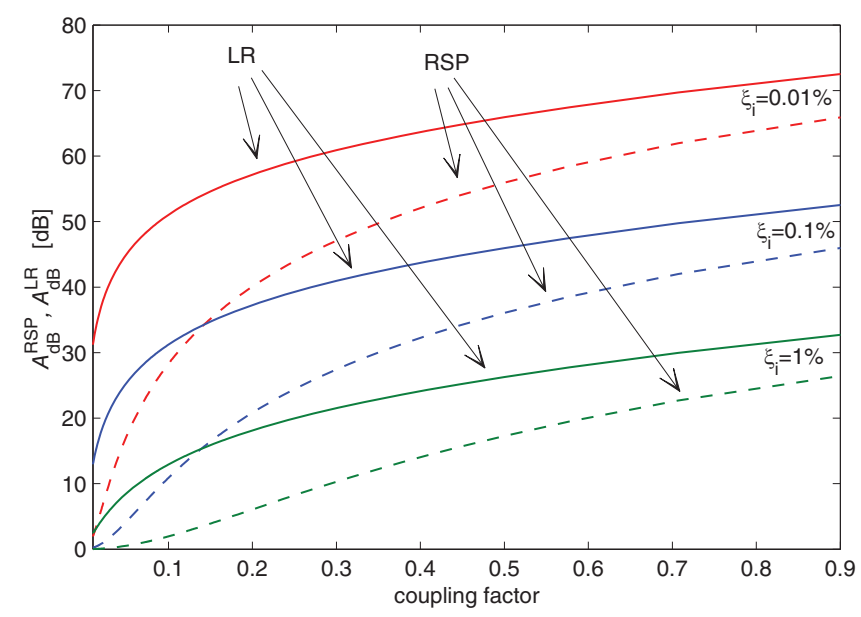

Figure 9. Trend of $A_{d \mathrm{~B}}^{R S P}$ and $A_{\mathrm{dB}}^{L R}$ as functions of the coupling factor: $k_{i}$ for the LR shunt and $\vec{k}_{i}$ for the RSP shunt.

\section{RSP and LR shunt performance comparisons}

This section compares the RSP and LR shunts in terms of attenuation performance when they are optimally tuned on the mechanical eigenmode to be damped (i.e. the values of $\tau_{e}, \omega_{e}$ and $\xi_{e}$ are the ones of Equations (13) and (23)).

Figure 9 shows the comparison between $A_{\mathrm{dB}}$ (Equation (14)) for the RSP (named $A_{\mathrm{dB}}^{R S P}$ from here on) and $A_{\mathrm{dB}}^{L R}$ (Equation (25)). Those two attenuation indexes are shown, for different values of $\xi_{i}$, as functions of the coupling factors: $k_{i}$ for the resonant shunt and $\tilde{k}_{i}$ for the RSP. One can remark that the higher the coupling factor is, the smaller the difference between the attenuations of the LR and RSP shunts is. Therefore, when the coupling factor is increased, the resistive shunt becomes more and more competitive with respect to the LR shunt.

However, in this case, the comparison between the two shunt techniques must not be carried out considering the same value of the coupling factor, i.e. $k_{i}=\tilde{k}_{i}$, since the resistive shunt has a higher coupling factor thanks to the use of the NCs in SP configuration (i.e. $\tilde{k}_{i}>k_{i}$ ). In light of this, Figure 10 is presented. It shows the trend of $A_{\mathrm{dB}}^{R S P}$ and $A_{\mathrm{dB}}^{L R}$ for different $G=\tilde{k}_{i} / k_{i}$ and $\xi_{i}$ values as function of $k_{i}$. The graph clearly shows that the use of the RSP becomes competitive (or even advantageous) if compared with LR shunt and this result is achieved thanks to the enhanced value of the MEMCF accomplished by using the SP.

To give another insight into the performance comparison between RSP and LR shunt, we define $k_{\text {lim }}$ as the value of $k_{i}$ for which $A_{d B}^{R S P}=A_{d B}^{L R}$ (i.e. defined in Figure 10 by the intersection of the dashed black curve with the others). Here $k_{\text {lim }}$ is by definition a function of $G$ and $\xi_{i}$. Figure 11 shows $k_{\text {lim }}$ as a function of $G$ for several values of $\xi_{i}$. One remarkable result is that the dependence of $k_{\text {lim }}$ on $\xi_{i}$ is almost negligible. Therefore,
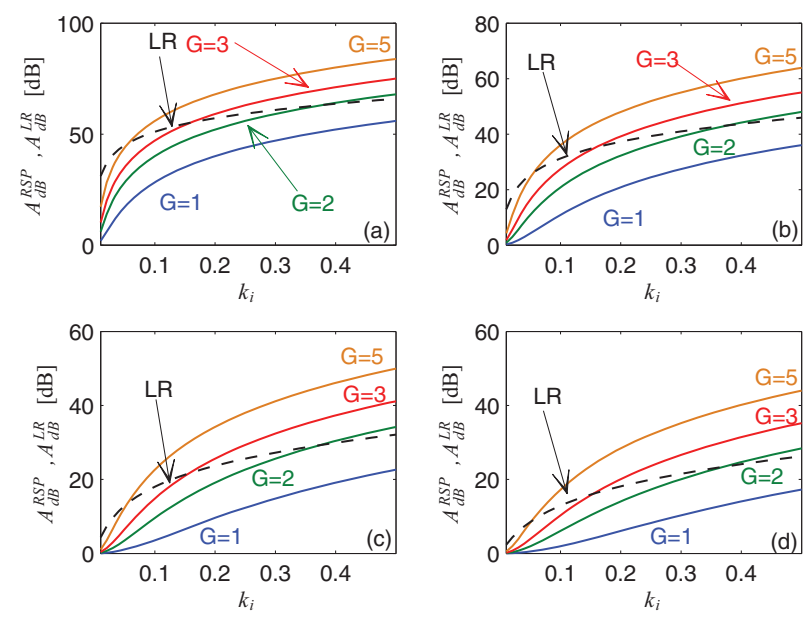

Figure 10. Trend of $A_{\mathrm{dB}}^{R S P}$ (coloured solid lines) and $A_{\mathrm{dB}}^{L R}$ (black dashed lines) as a function of $k_{i}$ for different values of $\xi_{i}$ : $\xi_{i}=10^{-4}(\mathrm{a}), \xi_{i}=10^{-3}(\mathrm{~b}), \xi_{i}=5 \times 10^{-3}(\mathrm{c})$ and $\xi_{i}=10^{-2}(\mathrm{~d})$.

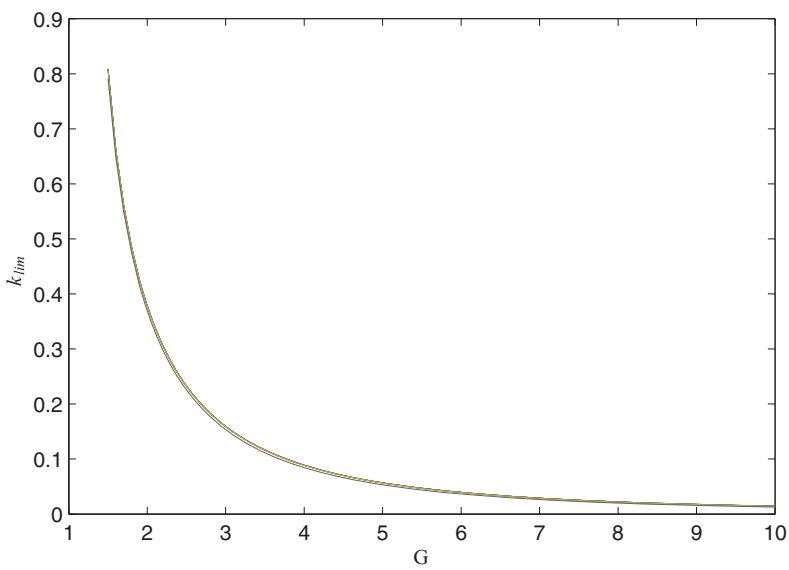

Figure II. Trend of $k_{\lim }$ as a function of $G$ for different values of $\xi_{i}$ (i.e. from $10^{-8}$ to $10^{-2}$ ). The lines are not plotted for $G$ values lower than $\mathrm{I} .5$ because $k_{\text {lim }}$ would be higher than I, which is not physical.

$k_{\text {lim }}$ can be considered a function of $G$ only: it depends just on the MEMCF enhancement due to the use of $\mathrm{NCs}$, whichever system is considered. As a consequence, Figure 11 plays an important role in the design process since it gives the relation between $G$ and $k_{i}$ for which the RSP shunt is as powerful as the traditional LR shunt. For instance, for values of $G$ and $k_{i}$ achievable in practical applications, such as $G=2.7$ (see further in the paper, i.e. the section related to the experimental tests) and $k_{i}$ greater than 0.2 (see Ducarne et al., 2012), the RSP shunt guarantees better performances than the traditional LR shunt.

\section{Robustness of LR shunt}

This section addresses the robustness of a LR shunt to a detuning of the electrical parameter, as a preliminary step towards its comparison with the behaviour of the 
(a)

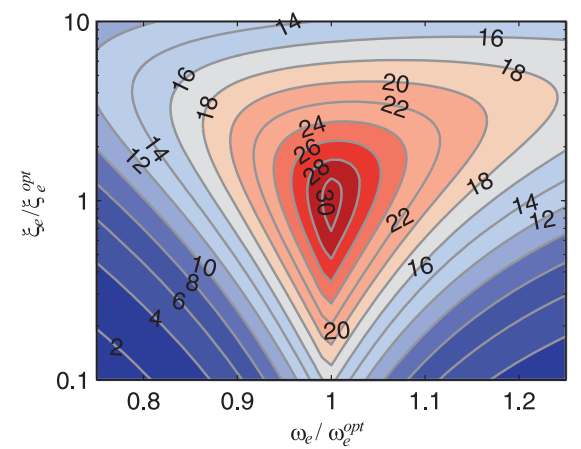

(c)

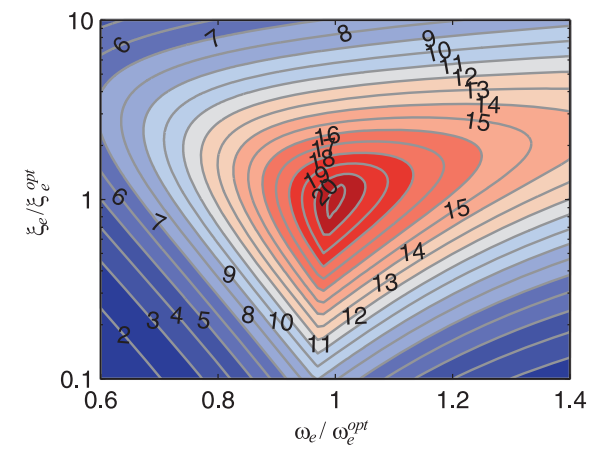

(b)

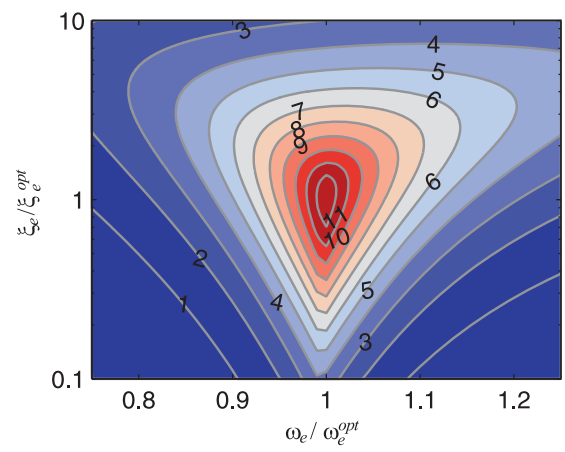

(d)

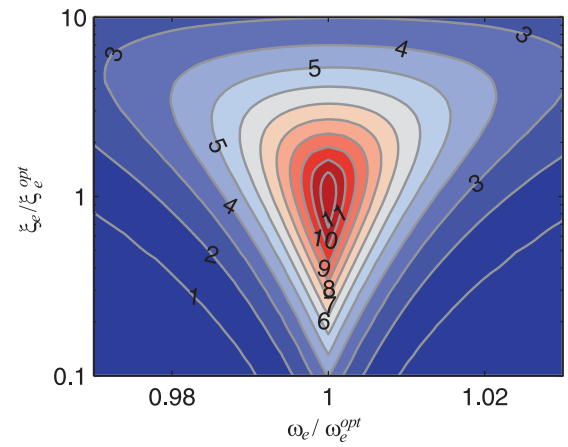

Figure 12. Attenuation $A_{\mathrm{dB}}^{* L R}$ provided by LR shunt in the presence of mistuning on the electrical parameters: (a) $\left(k_{i}, \xi_{i}\right)=\left(0.1,10^{-3}\right)$; (b) $\left(k_{i}, \xi_{i}\right)=\left(0.1,10^{-2}\right)$; (c) $\left(k_{i}, \xi_{i}\right)=\left(0.3,10^{-2}\right)$; (d) $\left(k_{i}, \xi_{i}\right)=\left(0.01,10^{-3}\right)$.

RSP shunt (see the next section). It is well known in the literature (Behrens et al., 2003; Berardengo et al., 2015a) that the traditional LR shunt undergoes significant attenuation losses when biases occur on the shunt parameters. As already done for the resistive shunt coupled to the SP layout, we analyse the effect of a mistuning of the shunt parameters on the performance in terms of vibration attenuation. Using Equation (24), we compute $A_{d B}^{* L R}$, defined by Equation (19), with $H^{\max }$ being the FRF peak of the EMS with the mistuned shunt, i.e. with $\omega_{e} \neq \omega_{e}^{o p t}$ and $\xi_{e} \neq \xi_{e}^{o p t}$. This represents a strict mistuning of the shunt, but also a change of the structural characteristics of the EMS since $\omega_{e} / \omega_{e}^{o p t}=\omega_{i} / \omega_{i}^{*}$, with $\omega_{i}^{*}$ the current value of the EMS natural frequency, different from its reference value $\omega_{i}$.

Figure 12 shows the attenuation performance $A_{d B}^{* L R}$ for LR shunt in the presence of mistuning, i.e. as a function of $\omega_{e} / \omega_{e}^{o p t}=\omega_{i} / \omega_{i}^{*}$ and $\xi_{e} / \xi_{e}^{o p t}$, for some systems chosen as examples (i.e. different values of $\xi_{i}$ and $k_{i}$ ). These figures show that the vibration attenuation is drastically reduced when the shunt impedance is not perfectly tuned on the mode which must be damped. We can also observe, for instance by looking at the limits of the axes on the plots, that the LR shunt is much less robust to a detuning on the electrical frequency (a change of $\omega_{e}$ ) than to a detuning of the electrical damping (a change of $\xi_{e}$ ).
Figure 13 shows the trend of $A_{\mathrm{dB}}^{* L R}-A_{\mathrm{dB}}^{L R}$ for the same cases investigated in Figure 12. The shapes of the plots of Figure 13 change according to the system considered. This means that the percentage loss of performance does not depend just on the percentage mistuning on $\omega_{e}$ and $\xi_{e}$, but also on the system considered. This is in contrast with what was evidenced previously for the enhanced resistive shunt. Indeed, in the case of RSP, all of the curves of Figure 8 are almost superimposed for attenuation levels over about $10 \mathrm{~dB}$. This meant that for RSP shunt the percentage loss of performance depends just on the percentage mistuning and not on the system considered.

These aspects related to the robustness of the LR shunt can be clearly inferred also by looking at Figures 14 and 15 , that show $A_{\mathrm{dB}}^{* L R}$ and $A_{\mathrm{dB}}^{* L R}-A_{\mathrm{dB}}^{L R}$ as a function of $\omega_{e} / \omega_{e}^{o p t}$ while the electrical damping is kept at its optimal value (i.e. $\xi_{e}=\xi_{e}^{o p t}$ ). The graphical comparison of Figures 14 and 15 to Figures 7 and 8 clearly shows the poor robustness of the LR shunt as compared with the R shunt (or RSP shunt).

\section{$R S P$ and $L R$ shunt robustness comparison}

In this section, the robustness of the two shunt impedances, RSP and LR, when changes in the EMS occur, is addressed. 
(a)

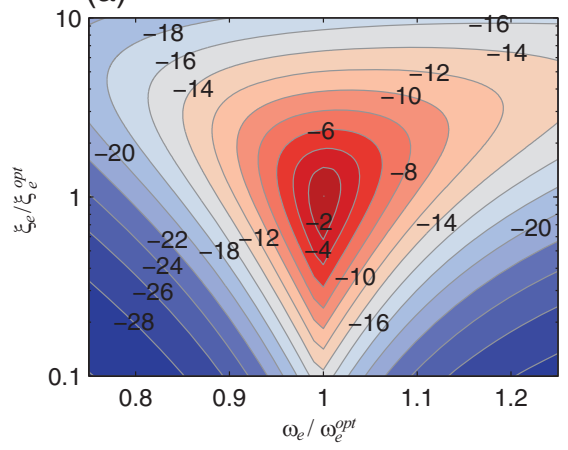

(c)

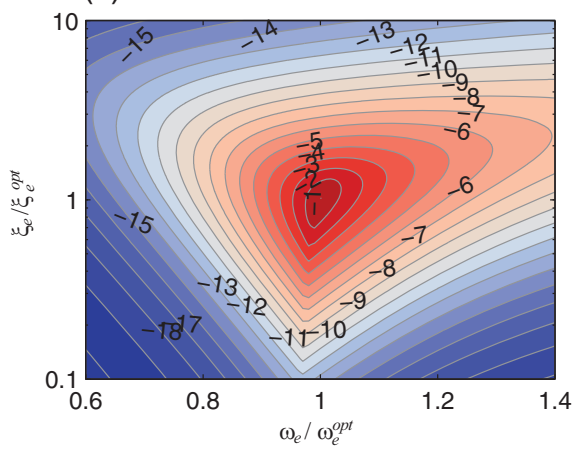

(b)

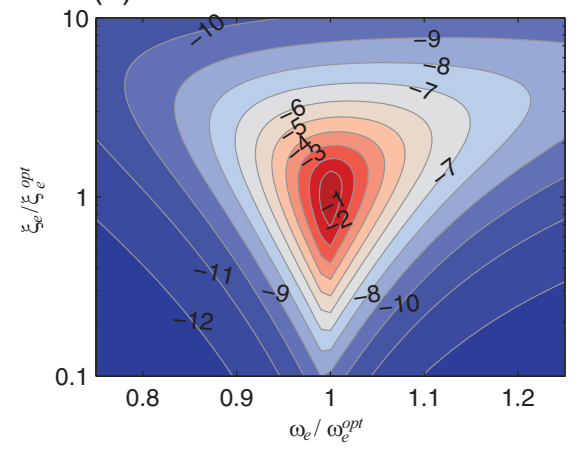

(d)

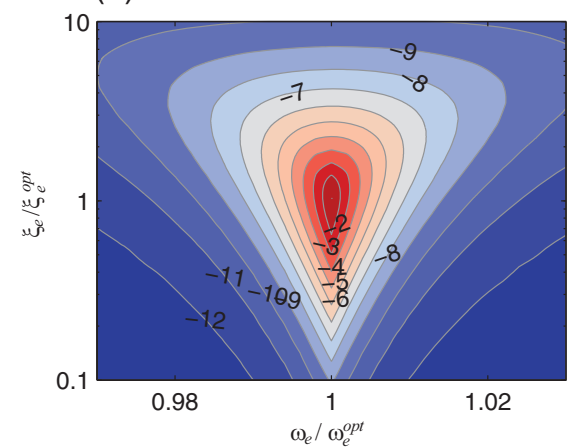

Figure 13. Trend of $A_{d B}^{* L R}-A_{d B}^{L R}$ provided by $L R$ shunt in the presence of mistuning on the electrical parameters: (a) $\left(k_{i}, \xi_{i}\right)=\left(0.1,10^{-3}\right) ;(\mathrm{b})\left(k_{i}, \xi_{i}\right)=\left(0.1,10^{-2}\right) ;(\mathrm{c})\left(k_{i}, \xi_{i}\right)=\left(0.3,10^{-2}\right) ;(\mathrm{d})\left(k_{i}, \xi_{i}\right)=\left(0.01,10^{-3}\right)$.
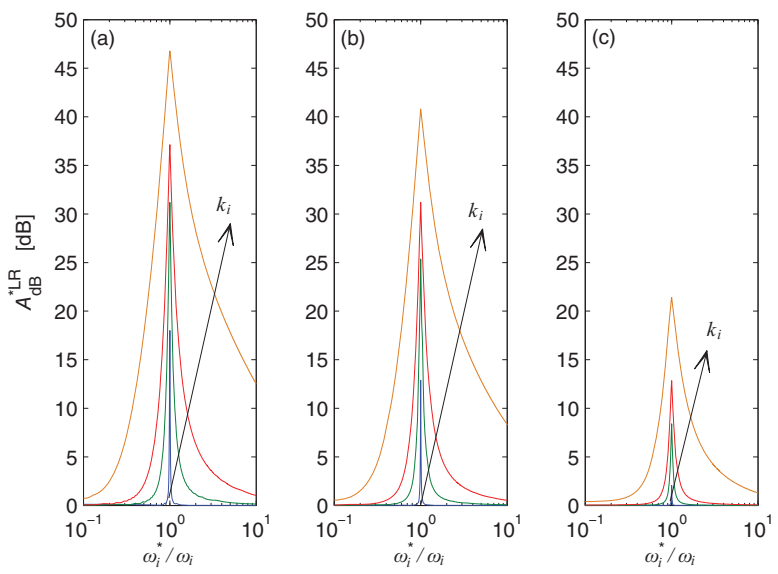

Figure 14. Trend of $A_{\mathrm{dB}}^{* L R}$ as a function of $\omega_{i}^{*} / \omega_{i}$ for different values of $k_{i}$ (i.e. $\left.0.01,0.05,0.1,0.3\right)$ for: $\xi_{i}=5 \times 10^{-4}(\mathrm{a})$, $\xi_{i}=10^{-3}(\mathrm{~b}), \xi_{i}=10^{-2}(\mathrm{c})$.

The robustness of the shunt methods is here analysed by supposing to start from perfectly tuned situations and then to experience a shift of the system eigenfrequency $\omega_{i}$ (e.g. due to a thermal shift). The shifted value of $\omega_{i}$ is defined here as $\omega_{i}^{*}$, as done previously when considering the robustness of the RSP and LR shunts. This natural frequency detuning affects the shunts tuning through $\tau_{e}$ for the RSP shunt $\left(\omega_{i}^{*} / \omega_{i}=\tau_{e} / \tau_{e}^{o p t}\right)$ and $\omega_{e}$ for the LR shunt $\left(\omega_{i} / \omega_{i}^{*}=\omega_{e} / \omega_{e}^{o p t}\right)$, while it does
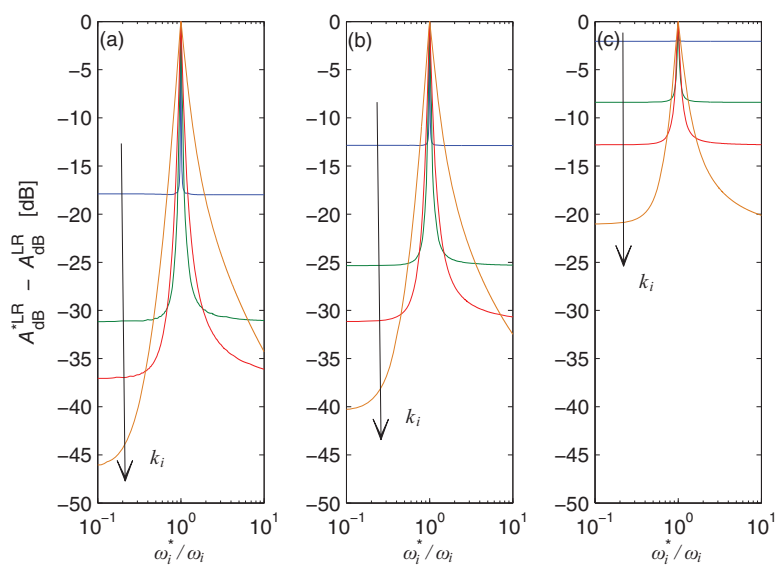

Figure 15. Trend of $A_{\mathrm{dB}}^{* L R}-A_{\mathrm{dB}}^{L R}$ as a function of $\omega_{i}^{*} / \omega_{i}$ for different values of $k_{i}$ (i.e. $0.01,0.05,0.1,0.3$ ) for: $\xi_{i}=5 \times 10^{-4}$ (a), $\xi_{i}=10^{-3}$ (b), $\xi_{i}=10^{-2}$ (c).

not affect the electrical damping $\xi_{e}$ of the LR shunt. Then, the difference $\Delta^{*}=A_{d B}^{* R S P}-A_{d B}^{* L R}$ (where $A_{d B}^{* R S P}$ is the attenuation in decibels in mistuned conditions for the RSP and it is calculated numerically by means of Equation (12)) has been computed for different values of $k_{i}$ and $\omega_{i}^{*} / \omega_{i}$, and taking into account three different values of $\xi_{i}$. This analysis has been repeated considering several values of the coupling factor enhancement $G=\tilde{k}_{i} / k_{i}$. 
(a)

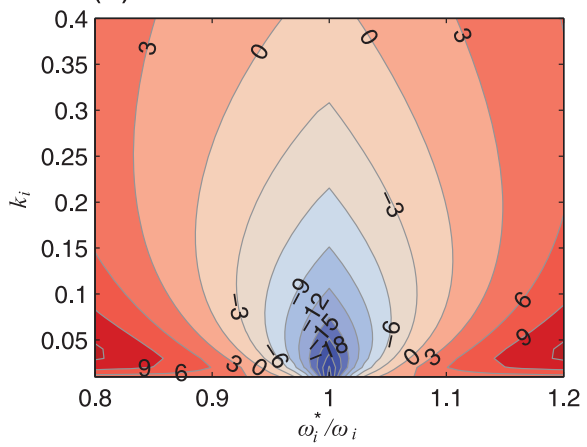

(c)

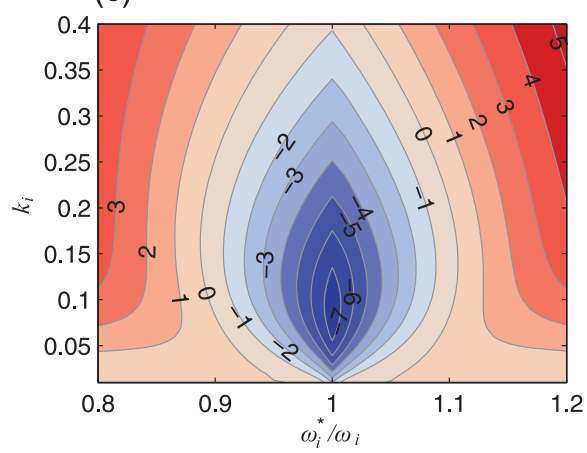

(b)

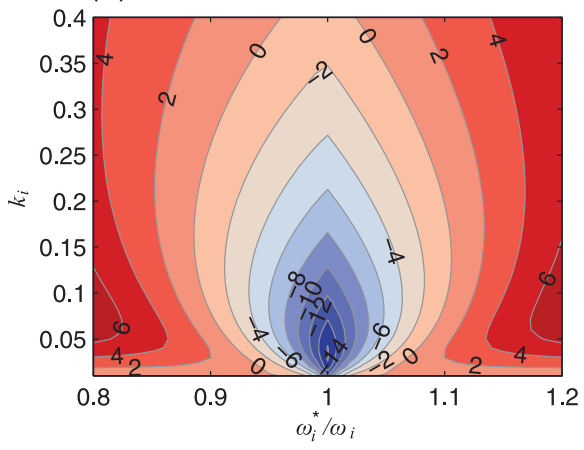

(d)

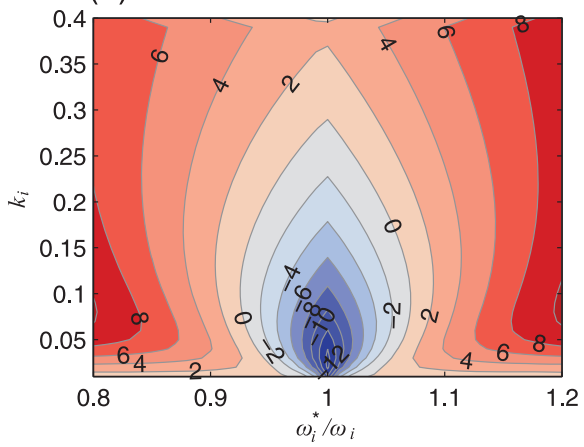

Figure 16. Trend of $\Delta^{*}$ as a function of $k_{i}$ and $\omega_{i}^{*} / \omega_{i}$ for different values of $\xi_{i}$ with: $G=2.38$ and $\xi_{i}=10^{-4}(\mathrm{a}), G=2.38$ and $\xi_{i}=10^{-3}(\mathrm{~b}), G=2.38$ and $\xi_{i}=10^{-2}(\mathrm{c}), G=3.00$ and $\xi_{i}=10^{-3}$ (d).

Figure 16(a)-(c) show the case where $\beta_{s p 1}=\beta_{s p 2}=0.7$, which in turn leads to a coupling factor enhancement $G$ equal to 2.38 . These maps highlight that the performance of the RSP shunt becomes more and more competitive as soon as the value of $k_{i}$ is increased and the mistuning increases (i.e. values of $\omega_{i}^{*} / \omega_{i}$ farther and farther from 1). Furthermore, if we increase the value of $G$ (compare Figures 16(c) and (d)), the area in which the RSP provides higher attenuation than the LR shunt (i.e. positive values of $\Delta^{*}$ ) increases as well.

This analysis showed that the RSP shunt is much more robust to biases and uncertainty than the pure resonant shunt. Therefore, the use of a resistive shunt coupled to a SP NC configuration is able to improve a lot the performances of simple resistive shunt, thus making it competitive with the LR shunt, keeping at the same time its high robustness.

\section{$R S P$ and $L R$ shunt comparison for broadband damping action}

In this section, the broadband control capability of the two circuits (RSP and LR) is analysed. Both R and LR shunts are well-known techniques for single mode control. Nevertheless, it can be deduced from the mechanical analogy with a damper (Preumont, 2011) that the $\mathrm{R}$ shunt is characterized by a wideband control action if compared with the resonant shunt. Indeed, the use of resistive shunt enables to have a significant control action even on the other modes of the EMS, while the resonant shunt works almost on the targeted mode. This can be easily demonstrated by taking into account the following case, as an example. Consider two modes of the same EMS, one at $\omega_{1}$ and the other at $\omega_{2}$ with $k_{1}=k_{2}$. The value of $\tau_{e}, \omega_{e}$ and $\xi_{e}$ are chosen to optimize the attenuation on mode 1 (see Equation (13) for the RSP and Equations (23) for the LR). Then, we compute $\Delta_{2}=A_{d B 2}^{R S P}-A_{d B 2}^{L R}$, where $A_{d B 2}^{R S P}$ and $A_{d B 2}^{L R}$ are the attenuations on the second mode, in decibels, for the RSP and LR shunt respectively and they are calculated numerically by means of Equations (12) and (24).

Figure 17 shows the attenuation difference on mode 2, i.e. $\Delta_{2}$, as function of the ratio $\omega_{2} / \omega_{1}$, for different values of $G$ (Figure 17(a)), $k_{i}$ (Figure 17(b)) and $\xi_{i}$ (Figure 17(c) and (d)). It is evident that the broadband action provided by the RSP shunt is much higher than that provided by the resonant shunt because its attenuation on mode 2 is much higher even for values of $\omega_{2} / \omega_{1}$ very low (i.e. close to 0.1 ) or very high (i.e. close to 10 ). The RSP shunt has thus shown to be a competitive alternative to the well-established LR shunt thanks to its higher robustness and its capability to damp many modes at the same time. Furthermore, the enhanced value of the MEMCF allows the RSP shunt to achieve maximum attenuations close (or higher) than the resonant shunt. 
Table 3. Modal data of the experimental set-up.

\begin{tabular}{llll}
\hline Mode & $\omega_{i} /(2 \pi)[\mathrm{Hz}]$ & $\xi_{i}$ & $k_{i}$ \\
\hline 1 & 39.72 & 0.0045 & 0.2716 \\
2 & 187.34 & 0.0030 & 0.1037 \\
3 & 509.00 & 0.0027 & 0.0710 \\
4 & 986.42 & 0.0028 & 0.1222 \\
\hline
\end{tabular}

\section{Experiments}

\section{Experimental set-up}

The RSP shunt has been tested experimentally to show its reliability and its capability to improve the attenuation performance, if compared with the resistive shunt coupled to the traditional $\mathrm{NC}$ in series and parallel configurations, and to validate the theoretical results shown so far. As for the comparison between the RSP and LR shunt, here no tests with the resonant shunt are shown because the related model has been already experimentally validated in Thomas et al. (2012) and its robustness tested in Berardengo et al. (2015a).

The experimental test set-up was a stainless steel cantilever beam (length of $178.8 \mathrm{~mm}$, width of $30.5 \mathrm{~mm}$ and thickness of $1.1 \mathrm{~mm}$ ) with two PIC 151 piezoelectric patches (with a length of $70 \mathrm{~mm}$ each, $0.5 \mathrm{~mm}$ of thickness and wrapped electrodes) bonded at the clamped end and electrically connected in series. The modes considered for the experimental activity were the first four bending modes, whose characteristics are given in Table 3 (the shapes of these modes are the usual ones for cantilever beams). Particularly, the values of $\omega_{i}$ and $\xi_{i}$ were estimated by means of experimental modal analysis, as well as the mode shapes, and the values of $k_{i}$ were estimated by deriving the $\omega_{i}$ and $\hat{\omega}_{i}$ values from the measured FRFs of the EMS in SC and $\mathrm{OC}$ and using Equation (10).

The value of the capacitance of the piezoelectric patch was measured at a number of frequency values between the first and the fifth mode of the structure with a digital multimeter. The obtained values of $C_{p i}$ were close each other: between 31.1 and $30.0 \mathrm{nF}$. Since these values are very close each other, we decided to use a sort of average value for all of the modes: $C_{\mathrm{p} i} \simeq C_{\mathrm{p}}=30.5 \mathrm{nF}$ for each of them $(i=1,2,3,4)$, denoted by $C_{\mathrm{p}}$ in the following. Here $C_{0}$ was estimated by measuring with the digital multimeter the value of $C_{\mathrm{p} 1}$ at $100 \mathrm{~Hz}$ and then calculating $C_{0}=C_{\mathrm{p} 1}\left(1+k_{1}^{2}\right)$ (see Berardengo et al., 2016). The result for $C_{0}$ was about $33.26 \mathrm{nF}$. Here $C_{\infty}$ was estimated measuring the capacitance of the piezoelectric patch at $100 \mathrm{kHz}$ and the result was about $25.9 \mathrm{nF}$.

The cantilever beam was excited by a contactless electromagnetic actuator, described in Thomas et al. (2003), composed by a fixed coil and a magnet bonded on the beam, close to its tip. The reference signal for
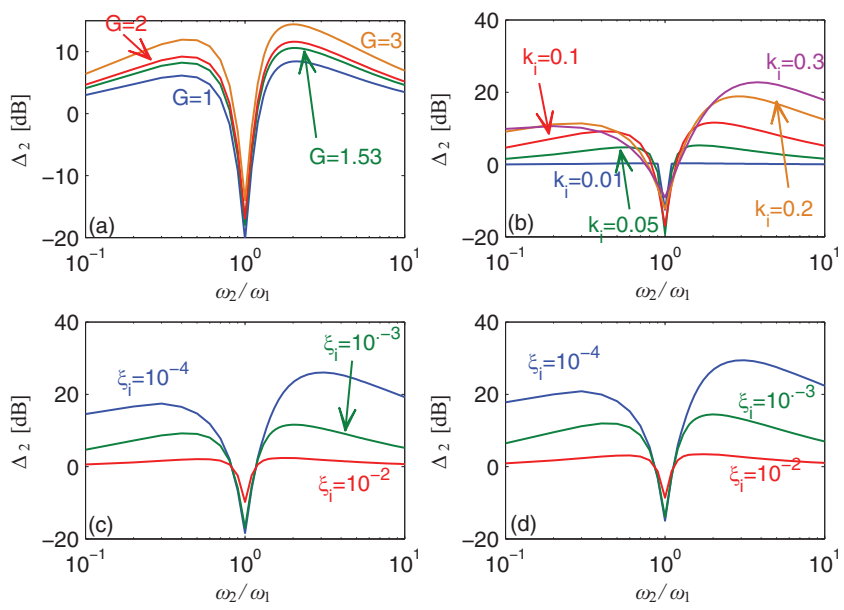

Figure I7. Trend of $\Delta_{2}$ as a function of $\omega_{2} / \omega_{1}$ for different values of $G$ with $k_{i}=0.1$ and $\xi_{i}=10^{-3}(\mathrm{a})$, for different values of $k_{i}$ with $G=2$ and $\xi_{i}=10^{-3}(\mathrm{~b})$, for different values of $\xi_{i}$ for $k_{i}=0.1$ and $G=2(\mathrm{c})$, for different values of $\xi_{i}$ for $k_{i}=0.1$ and $G=3(d)$.

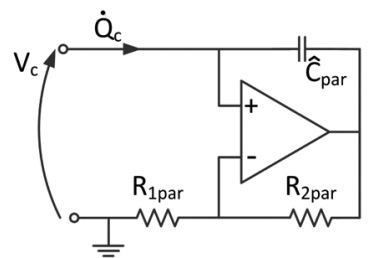

(a)

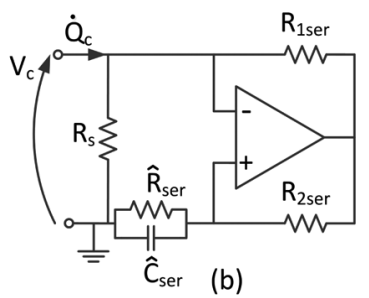

(b)
Figure 18. Negative capacitance practical layout using operational amplifiers: parallel NC (a) and series NC (b).

the FRF computation was the intensity of the current which flowed in the coil, measured by a current clamp (Tektronix A622), assumed proportional to the electromagnetic force imposed on the magnet. A Brüel \& Kjaer 2712 power amplifier was used to feed the coil. The response of the beam was measured by a laser Doppler velocimeter (Polytec PSV 400). The NCs were built as proposed in Figures 18 and 19 using Texas Instruments OPA445 operational amplifiers (TexasInstruments, 2010), with some of the resistances built using potentiometers for easy tuning.

It should be noted that the NCs in parallel can be considered as pure NCs:

$$
-C_{1}=-\frac{R_{2 p a r} \hat{C}_{p a r}}{R_{1 p a r}}
$$

Conversely, the NCs in series cannot be considered as pure NCs because of the presence of the resistance $\hat{R}_{s e r}$. This latter component must be added in parallel to the capacitance $\hat{C}_{\text {ser }}$ to form together with $\hat{C}_{\text {ser }}$ an undesired but necessary high-pass filter used to solve practical problems such as bias-current- and offset-voltageinduced errors (Moheimani and Fleming, 2006). 


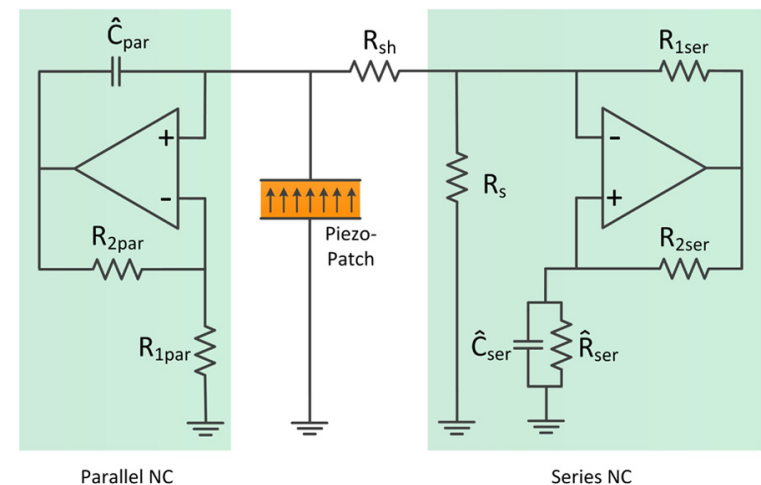

Figure 19. Scheme of the electric shunt for the SP configuration, using two operational amplifiers.

Therefore, the circuit of Figure 18(b) and the series part of Figure 19 composed by $\hat{R}_{s e r}, \hat{C}_{s e r}, R_{1 s e r}$ and $R_{2 s e r}$ can be seen as the parallel of a NC and a negative resistance $-\tilde{R}$ :

$$
-C_{2}=-\frac{R_{2 s e r} \hat{C}_{s e r}}{R_{1 s e r}}, \quad-\tilde{R}=-\frac{R_{1 s e r} \hat{R}_{s e r}}{R_{2 s e r}}
$$

In order to limit the effect on the circuit behaviour of this negative resistance $-\tilde{R}$ in parallel to the NC, a further resistance $R_{s}$ is added in the circuits of the NCs in series. This further element is indeed used to compensate for the effects of the negative resistance $-\tilde{R}$, almost cancelling its effects. Indeed, the global resistance $R_{e q}$ given by the parallel between $R_{S}$ and $-\tilde{R}$ is:

$$
R_{e q}=\frac{-\tilde{R} R_{S}}{R_{s}-\tilde{R}}
$$

If the value of $R_{s}$ is chosen so that the value of $R_{e q}$ is negative and as high as possible in terms of absolute value, the impedance made up by $R_{S},-\tilde{R}$ and $-C_{2}$ approximates a pure NC. More details about the use of $R_{s}$ for NCs in series can be found in Berardengo et al. (2016); the same referenced paper also gives details about the possible use of a resistance in parallel to $\hat{C}_{p a r}$ for NCs in parallel configuration (see Figure 18(a)).

Many tests were carried out to show the benefits provided by the RSP if compared with the series and parallel resistive shunt. Two of them are discussed in the following.

\section{Tests}

Here two different tests are taken into account. The first, denoted as Test1, is related to a case in which $\beta_{1}=\beta_{s p 1}=0.65$ and $\beta_{2}=\beta_{s p 2}=0.82$ were used. The second test considered here, named Test2, has instead the following configuration: $\beta_{1}=\beta_{s p 1}=0.65$ and $\beta_{2}=\beta_{s p 2}=0.61$. In both tests, NCs in parallel, series and SP configurations, coupled to a resistive shunt,
Table 4. Values of the electric components used in Testl and Test2.

\begin{tabular}{lll}
\hline & Testl & Test2 \\
\hline$C_{1}[\mathrm{nF}]$ & 19.82 & 19.82 \\
$C_{2}[\mathrm{nF}]$ & 37.21 (series), & 50.00 (series), \\
& 17.39 (SP) & 30.18 (SP) \\
$R_{s}[\mathrm{M} \Omega]$ & 2.87 & 2.87 \\
$R_{\text {Ipar }}[\mathrm{k} \Omega]$ & 4.64 & 4.64 \\
$R_{2 \text { par }}[\mathrm{k} \Omega]$ & 4.64 & 4.64 \\
$R_{\text {Iser }}[\mathrm{k} \Omega]$ & 7.24 (series), & 5.48 (series), \\
$R_{2 \text { ser }}[\mathrm{k} \Omega]$ & 15.18 (SP) & 9.06 (SP) \\
$\hat{C}_{\text {par }}[\mathrm{nF}]$ & 4.64 & 4.64 \\
$\hat{C}_{\text {ser }}[\mathrm{nF}]$ & 19.82 & 19.82 \\
$\hat{R}_{\text {ser }}[\mathrm{M} \Omega]$ & 58.05 (series), & 59.05 (series), \\
& 58.93 (SP) & 51.81 (SP) \\
\hline
\end{tabular}

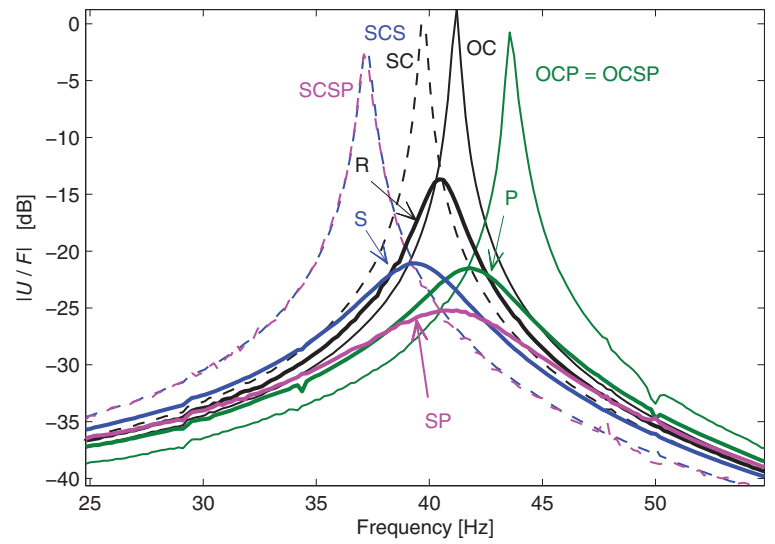

Figure 20. Experimental FRFs (displacement $U$ to force $F$ ratio) for Test2 using the optimal value of $\tau_{\mathrm{e}}$ for the first mode: zoom on mode I. Letter $S$ indicates the FRF in series with the optimal value of $\tau_{\mathrm{e}}, \mathrm{P}$ the optimal parallel, SP the optimal series + parallel. SCSP indicates the short-circuit FRF for the SP, SCS the short-circuit FRF for the series, OCP the open-circuit FRF for the parallel and OCSP the open-circuit FRF for the SP. R indicates the optimal resistive shunt without NCs.

have been investigated; in addition, we tested also the pure resistive shunt without NCs. The $\tau_{e}$ values have been fixed equal to $\tau_{e}^{o p t}$ of the first and fourth modes for both the tests and all the three NC configurations. The values of the electric components for the tests are gathered in Table 4. It is remarked that the $\beta$ values of Test 1 were chosen in order to provide the best attenuation value and at the same time a reliable functioning of the electrical impedance.

Figure 20 shows the FRFs (zoomed on the first mode) with the value of $\tau_{e}$ optimized for the damping of the first mode for each NC configuration for Test 2 . Figure 21 shows the FRFs (zoomed on the third mode) with the value of $\tau_{e}$ optimized for the damping of the 
Table 5. Comparison between experimental and theoretical attenuations for Testl.

\begin{tabular}{|c|c|c|c|c|c|}
\hline & & \multicolumn{4}{|c|}{ Attenuation [dB] Experimental - Numerical } \\
\hline & & Mode I & Mode 2 & Mode 3 & Mode 4 \\
\hline \multirow[t]{3}{*}{ Tuning on mode I } & Parallel & $21.8-22.1$ & $6.2-8.1$ & $2.0-1.8$ & $2.2-2.3$ \\
\hline & Series & $27.1-27.0$ & $8.9-8.4$ & $2.6-2.6$ & $3.4-3.3$ \\
\hline & & $29.3-29.6$ & $11.6-10.2$ & $3.6-3.5$ & $4.4-4.4$ \\
\hline \multirow[t]{3}{*}{ Tuning on mode 4} & Parallel & $5.5-5.5$ & $5.3-5.5$ & $6.4-6.4$ & $13.4-13.7$ \\
\hline & Series & $10.3-7.3$ & $5.1-8.3$ & $9.8-9.6$ & $16.3-18.1$ \\
\hline & SP & $12.1-9.1$ & $7.2-9.9$ & $11.7-11.4$ & $19.3-20.4$ \\
\hline
\end{tabular}

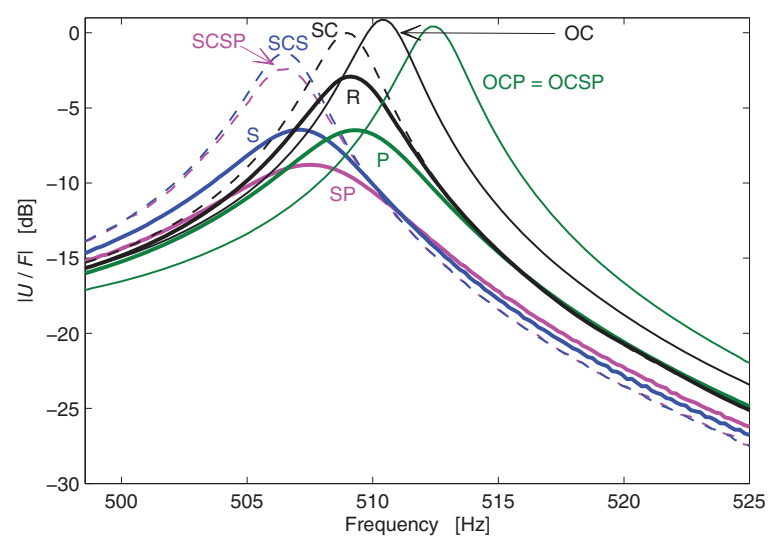

Figure 21. Experimental FRFs (displacement $U$ to force $F$ ratio) for Test2 using the optimal value of $\tau_{\mathrm{e}}$ for the fourth mode: zoom on mode 3. Letter $S$ indicates the FRF in series with the optimal value of $\tau_{\mathrm{e}}, \mathrm{P}$ the optimal parallel, SP the optimal series + parallel. SCSP indicates the short-circuit FRF for the SP, SCS the short-circuit FRF for the series, OCP the open-circuit FRF for the parallel and OCSP the open-circuit FRF for the SP. R indicates the optimal resistive shunt without NCs.

fourth mode for each NC configuration for Test2. In both cases, the increased attenuation provided by the $\mathrm{SP}$ is evident, as well as its capability to shift both the $\mathrm{SC}$ and $\mathrm{OC}$ eigenfrequencies (as already explained in the paper). It is remarked that the SP layout improves the attenuation both in tuned (Figure 20) and mistuned (Figure 21) conditions. Moreover, Figure 21 highlights also the broadband control capability of the resistive shunt, which is further enhanced by the use of RSP configuration.

Figures 22 and 23 show the comparison between numerical and experimental FRFs of Test 1 for $\tau_{e}$ tuned on the first and fourth mode, respectively. These plots evidence that the analytical model used herein is able to accurately describe the experimental evidence.

Finally, Tables 5 and 6 summarize the attenuations on all of the four modes for all of the tests presented here, showing again the benefits provided by the SP. Indeed, the SP is able to increase the attenuation if compared with the series and parallel layouts both in tuned and mistuned conditions (for this latter case refer

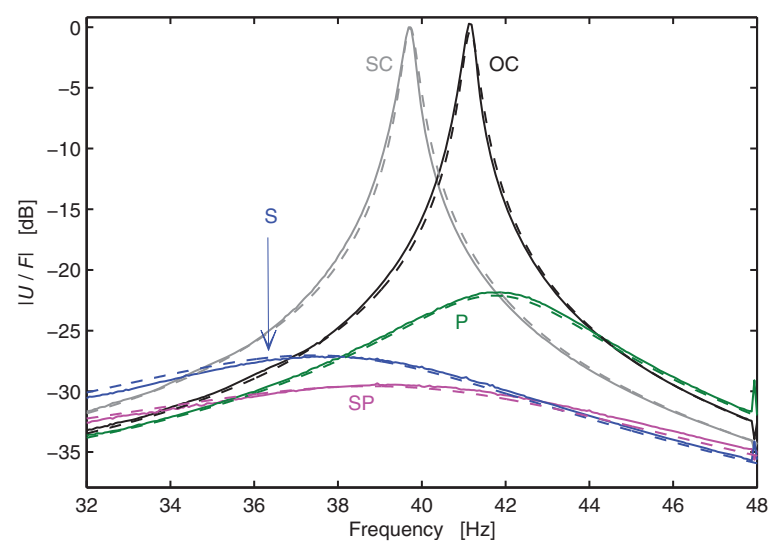

Figure 22. Comparison between the experimental (solid lines) and analytical (dashed lines) FRFs (displacement $U$ to force $F$ ratio) for Test I and $\tau_{\mathrm{e}}$ tuned on the first mode around mode $\mathrm{I}$.

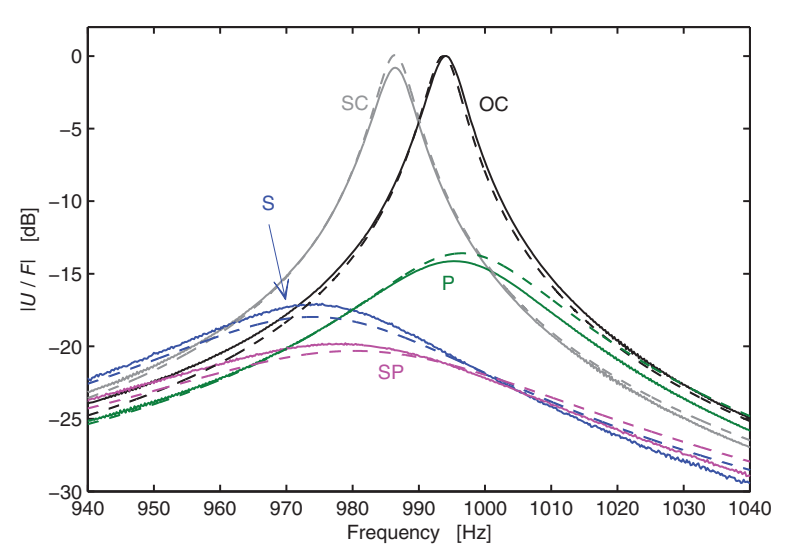

Figure 23. Comparison between the experimental (solid lines) and analytical (dashed lines) FRFs (displacement $U$ to force $F$ ratio) for Testl and $\tau_{\mathrm{e}}$ tuned on the fourth mode around mode 4 .

to the results achieved for the modes different from that on which the value of $\tau_{e}$ has been tuned). This is made possible by the increased values of $G$ provided by the SP layout, which are gathered in Table 7. Furthermore, the RSP shunt is expected to be more robust than a LR shunt, providing on the first mode almost the same 
Table 6. Comparison between experimental and theoretical attenuations for Test2.

\begin{tabular}{|c|c|c|c|c|c|}
\hline & & \multicolumn{4}{|c|}{ Attenuation $[\mathrm{dB}]$ Experimental - Numerical } \\
\hline & & Mode I & Mode 2 & Mode 3 & Mode 4 \\
\hline \multirow[t]{3}{*}{ Tuning on mode I } & Parallel & $21.5-22.1$ & $8.0-8.1$ & $1.4-1.8$ & $2.5-2.3$ \\
\hline & Series & $2.1-2.3$ & $6.2-5.7$ & $1.6-1.5$ & $2.3-2.1$ \\
\hline & & $25.2-25.7$ & $9.7-8.4$ & $2.3-2.5$ & $4.0-3.5$ \\
\hline \multirow[t]{3}{*}{ Tuning on mode 4} & Parallel & $5.6-5.5$ & $5.3-5.5$ & $6.5-6.4$ & $13.4-13.7$ \\
\hline & Series & $6.4-4.9$ & $4.1-5.2$ & $6.4-5.9$ & $12.1-12.9$ \\
\hline & SP & $8.6-7.3$ & $6.2-7.7$ & 8.8. - 8.5 & $16.1-16.8$ \\
\hline
\end{tabular}

Table 7. The $G$ values for Testl and Test 2 for the parallel, series and SP layouts.

\begin{tabular}{llll}
\hline & \multicolumn{2}{l}{ Value of $G$} & \\
\cline { 2 - 4 } & Parallel & Series & SP \\
\hline Testl & 1.69 & 2.35 & 2.72 \\
Test2 & 1.69 & 1.60 & 2.10 \\
\hline
\end{tabular}

attenuation in tuned conditions (see Figure 10(c)) for Test 2 configuration, and even higher for Test 1 .

\section{Conclusion}

This paper has dealt with the use of a new layout for NCs shunted to piezoelectric actuators for vibration damping. This configuration relies on the use of two $\mathrm{NCs}$, one connected in parallel and the other connected in series to the piezoelectric actuator. This layout (named SP), when coupled to a resistive shunt, is able to increase the attenuation performance provided by the traditional layouts (i.e. a single $\mathrm{NC}$ in either parallel or series). The paper has addressed all of the advantages provided by this new layout coupled to resistive shunt (called RSP shunt). Indeed, in the first part of the paper, it has been shown that the SP layout enables us to increase the stability margin for a given performance level, thus improving the reliability of the system. It is also shown quantitatively that the loss of performance of the shunt due to a mistuning (a change in the shunt parameters or in the structural characteristics of the host structure) is decreased with this new SP layout, thus proving the associated increase of robustness. In the second part, the RSP shunt performances are systematically compared with those of the traditional resonant shunt. It has been shown that the RSP shunt can bring to equivalent performances in term of vibration level attenuation than a resonant shunt, even for lightly damped host structures $\left(\xi_{i}<5 \times 10^{-3}\right)$ and standard modal electromechanical coupling factors $\left(k_{i}<0.2\right)$. At the same time, increased robustness to a detuning and broadband action have been demonstrated, as compared with the traditional resonant shunt, thus proving that the new RSP shunt is promising.

\section{Acknowledgment}

The authors would like to gratefully thank the Structural Mechanics and Coupled Systems Laboratory (LMSSCCnam, Paris) and its head Jean-François Deü for the loan of the piezoelectric beam used in the experiments.

\section{Declaration of Conflicting Interests}

The author(s) declared no potential conflicts of interest with respect to the research, authorship, and/or publication of this article.

\section{Funding}

The author(s) received no financial support for the research, authorship, and/or publication of this article.

\section{References}

Beck BS, Cunefare KA and Collet M (2013) The power output and efficiency of a negative capacitance shunt for vibration control of a flexural system. Smart Materials and Structures 22(6): 065009.

Beck BS, Cunefare KA and Collet M (2014) Response-based tuning of a negative capacitance shunt for vibration control. Journal of Intelligent Material Systems and Structures 25(13): 1585-1595.

Behrens S, Fleming AJ and Moheimani SOR (2003) A broadband controller for shunt piezoelectric damping of structural vibration. Smart Materials and Structures 12(1): $18-28$.

Berardengo M, Cigada A, Manzoni S and Vanali M (2015a) Vibration control by means of piezoelectric actuators shunted with LR impedances: Performance and robustness analysis. Shock and Vibration 2015: 704265.

Berardengo M, Manzoni S, Thomas $\mathrm{O}$ and Giraud-Audine C (2015b) A new electrical circuit with negative capacitances to enhance resistive shunt damping. In: Proceedings of the ASME 2015 Conference on Smart Materials, Adaptive Structures and Intelligent Systems (SMASIS 2015), Colorado Springs, CO, 21-23 September 2015.

Berardengo M, Thomas O, Giraud-Audine $\mathrm{C}$ and Manzoni S (2016) Improved resistive shunt by means of negative 
capacitance: new circuit, performances and multi modecontrol. Smart Materials and Structures 25: 075033.

Burns R (2001) Advanced control engineering. London: Butterworth-Heinemann.

Collet M, Ouisse M, Cunefare K, Ruzzene M, Beck B, Airoldi L and Casadei F (2011) Vibroacoustic energy diffusion optimisation in beams and plates by means of distributed shunted piezoelectric patches. In: Vasques $\mathrm{C}$ and Rodrigues JD (eds.) Vibration and structural acoustics analysis: current research and related technologies. Dordrecht: Springer, pp. 265-302.

Date M, Kutani M and Sakai S (2000) Electrically controlled elasticity utilizing piezoelectric coupling. Journal of Applied Physics 87(2): 863-868.

de Marneffe B and Preumont A (2008) Vibration damping with negative capacitance shunts: theory and experiment. Smart Materials And Structures 17(3): 035015.

Ducarne J, Thomas O and Deü J (2012) Placement and dimension optimization of shunted piezoelectric patches for vibration reduction. Journal of Sound and Vibration 331(14): 3286-3303.

Gopal M (2002) Control Systems: Principles and Design, 2nd edn.Tata McGraw-Hill Education.

Hagood N and von Flotow A (1991) Damping of structural vibrations with piezoelectric materials and passive electrical networks. Journal of Sound and Vibration 146: 243-268.

Heuss O, Solloum R, Mayer D and Melz T (2014) Tuning of a vibration absorber with shunted piezoelectric transducers. Archive of Applied Mechanics. DOI: 10.1007/s00419014-0972-5.

Horowitz P and Hill W (1989) The art of electronics, 2nd edn. Cambridge: Cambridge University Press.

Kodejška M, Mokrý P, Linhart V, Václavik $\mathbf{J}$ and Sluka T (2012) Adaptive vibration suppression system: An iterative control law for a piezoelectric actuator shunted by a negative capacitor. IEEE Transactions on Ultrasonics, Ferroelectrics, and Frequency Control 59(12): 2785-2796.

Manzoni S, Moschini S, Redaelli M and Vanali M (2012) Vibration attenuation by means of piezoelectric transducer shunted to synthetic negative capacitance. Journal of Sound and Vibration 331(21): 4644-4657.

Moheimani S and Fleming A (2006) Piezoelectric Transducers for Vibration Control and Damping. Berlin: SpringerVerlag.

Park CH and Baz A (2005) Vibration control of beams with negative capacitive shunting of interdigital electrode piezoceramics. Journal of Vibration and Control 11(3): 331-346.

Preumont A (2011) Vibration Control of Active Structures, 3rd edn. New York: Springer.

TexasInstruments (2010) OPA445, High Voltage FET-Input Operational Amplifier. Available at: http://www.ti.com/ product/opa445.

Thomas O, Deü JF and Ducarne J (2009) Vibration of an elastic structure with shunted piezoelectric patches: efficient finite-element formulation and electromechanical coupling coefficients. International Journal of Numerical Methods in Engineering 80(2): 235-268.

Thomas O, Ducarne J and Deü J (2012) Performance of piezoelectric shunts for vibration reduction. Smart Materials and Structures 21(1): 015008.

Thomas O, Touzé C and Chaigne A (2003) Asymmetric nonlinear forced vibrations of free-edge circular plates. Part II: experiments. Journal of Sound and Vibration 265(5): 1075-1101.

Yamada K, Matsuhisa H, Utsuno H and Sawada K (2010) Optimum tuning of series and parallel LR circuits for passive vibration suppression using piezoelectric elements. Journal of Sound and Vibration 329(24): 5036-5057. 